\title{
RESEARCH
}

Open Access

\section{Efficient energy resource utilization in a wireless sensor system for monitoring water quality}

Segun O. Olatinwo * (D) and Trudi H. Joubert

\begin{abstract}
In this paper, a new approach to energy harvesting and data transmission optimization in a heterogeneous-based multi-class and multiple resource wireless transmission wireless sensor network system that focus on monitoring water and its quality is presented. Currently, energy is a scarce resource in wireless sensor networks due to the limited energy budget of batteries, which are typically employed for powering sensors. Once the available energy of a particular sensor node battery is depleted, such sensor node becomes inactive in a network. As a consequence, such node may not be able to participate in the transmission of the application signal in the uplink stage of the network, resulting in a lack of ability to communicate vital signals in a timely manner. Energy scarcity has been a long standing problem in wireless sensor network applications. To address this problem, energy harvesting from intended radiofrequency power source is considered in this work. However, wirelessly powered wireless sensor network systems are confronted by unfairness in resource allocation problem, as well as interference problem in multiple energy resource transmissions. These problems adversely impact the performance of the system in the context of the harvested energy by the sensor nodes, sensors information transmission rates, and the overall system throughput rate. These problems are tackled in this paper by formulating a sum-throughput maximization problem to reduce system energy consumption and enhance the system overall throughput rate. The throughput optimization problem is formulated as a non-convex function. Through the exploitation of the problem structure, it is converted to a convex function. The mathematical models of the optimization problem are validated through numerical simulations. The simulation results reveal that the proposed wireless powered sensor network system outperforms an existing wireless powered sensor network system, by comparison of the numerical simulations of this work to the numerical simulations of the existing WPSN system, regardless of the distances of the sensor nodes to the IPS and the base station. Also, the newly proposed method performs efficiently using parameters that include path-loss exponent impact, performance comparison of systems, convergence based on iteration, comparison based on unequal network distances to the BS, transmission power impact on the attainable throughput and on fraction of energy consumed on information transmissions, and influence of different number of nodes in the network classes.
\end{abstract}

Keywords: Energy scarcity, Monitoring of water quality, Optimization, Resource allocation, Wireless sensor network

\footnotetext{
* Correspondence: segunlatinwo@gmail.com

Department of Electrical, Electronic and Computer Engineering, University of

Pretoria, Pretoria 0001, South Africa
} 


\section{Introduction}

In recent times, there has been an upsurge in the need for efficient sensing systems for monitoring the parameters of water quality that include bacteria load and $\mathrm{pH}$ values, in a timely fashion $[1,2]$. Most times, Escherichia coli is considered as an indicator organism for microbiological analysis of water [3-5]. The main reason for the upsurge in seeking efficient sensing systems is because of the devastating impact of unclean water on human, plant, and animal. The systems are intended to complement the existing traditional systems for effective monitoring of water and its quality, in order to combat the problem of contamination in water $[1,2,6]$. Across the globe, an approximate estimate of 250 million cases of disease caused by polluted water is reported annually [7]. These diseases are responsible for human death and claims up to about ten million lives across the globe annually [7]. This is an indication that water problems caused by contaminants are major issues in this dispensation. The alarming rate of human death on a global scale caused by water pollution is as a result of a surge in water and environmental contaminants. These contaminants are due to two major factors, namely natural processes and man-made (anthropogenic) activities [2, 8, 9]. Examples of natural phenomena that adversely influence the quality of water through climatic factors are run-off caused by hydrological conditions, rock weathering, soil leaching, depositions caused by wind, and evapotranspiration, while some of the key man-made activities that negatively influences the quality of water are mining operations, deforestation, agricultural run-off, and industrial effluent [10].

As a result of the surge in water contaminants, water consumed from either the water polluted by natural processes or man-made activities is dangerous to humans and the ecosystem, because of their high levels of heavy metals and microbes. The microbes and heavy metals cause havoc to human health. Examples of some of the disruptions they cause are diarrhea, epigastric pain, organ damage such as renal and hepatic failure, and cancer $[11,12]$. For example, around 1,500,000 children die due to diarrhea every year [13]. Heavy metals are highly toxic and also create a lot of environmental concerns [2, 14-16]. To address these issues, there is an urgent need for effective systems for frequently monitoring water quality parameters. To achieve this, the adoption of wireless sensor networks (WSNs) technology has been proposed as a promising solution. Unfortunately, WSNs are faced with several challenges that range from energy, memory, and processing capability. As a result of these limitations, both academia and industry are currently making efforts toward seeking solutions to the aforementioned problems. Among the issues raised in WSNs, the energy scarcity problem is the greatest of all, as the operation of other modules depends on energy [17, 18]. The problem of energy scarcity in WSNs has been in existence for a long time on the account of the limited energy budget of the batteries that are typically used for powering the sensor nodes in WSN systems [2, 19]. To meet the objective of WSN-based water quality monitoring systems in the context of timely monitoring without interruption in energy supply, harvesting energy from energy sources, which is technically referred to as energy harvesting $(\mathrm{EH})$ technique in practice is a promising approach $[2,20]$. The technique has been exploited by the energy harvesting research community in WSNs to replace the utilization of battery power, which is associated with several problems that include short life span, cost of battery replacement, and difficulty in battery replacement. Energy harvesting from sources that include solar $[2,21]$, radio frequency (RF) signals $[2,22,23]$, and wind [24] have been exploited. However, the most interesting energy source among the above-mentioned sources is RF EH from intentionally stationed power sources [2]. The main reason for this is that $\mathrm{EH}$ from intended RF power sources (IPS) is controllable, as well as suitable for continuous monitoring of water distribution networks [25]. Similarly, EH from IPS is suitable for energy transmissions over larger areas because of its far-field characteristics of energy radiation [25]. As a consequence of the benefits of EH from IPS, it is an attractive energy solution for water monitoring applications in enclosed environments, although the energy solution can be employed in any location.

At the moment, there are commercial IPS solutions [26]. This development is as a result of the advances in wireless energy transmission technology. One of the leading energy solution providers is Powercaster ${ }^{\oplus}$. Forms of RF transmitters from Powercaster ${ }^{\ominus}$ are the battery-powered IPS [27], and the TX91501 IPS [27]. They are reliable solution for transferring RF energy in a wireless manner and they can cover a distance of about $24 \mathrm{~m}$. They are compatible with the unlicensed bands of the industrial, scientific, and medical (ISM) model for communications [28]. In addition, the Powercaster ${ }^{\ominus}$ energy solutions come with a compatible RF harvester, which is employed at the sensor node for energy harvesting.

Recently, an RF EH method based on wireless energy transmission and wireless information transmission (WIPT) has emerged as a promising solution for powering sensor nodes in WSNs. The technique is suitable for conveying the signals of the sensor nodes to a local base station. Such a system is typically referred to as a sensor network that is based on wireless powering (WPSN). With this WIPT technique, abundant RF energy could be efficiently transferred from an IPS to a large number of sensor nodes in a network to achieve a stable supply of energy without any interruption in communication 
that may result due to energy depletion [25, 29]. This method employs harvesting of RF energy from an IPS, compared to the traditional EH methods that harnesses energy from ambient energy sources, which may include ambient RF. This WIPT method is envisioned to provide a lasting solution to the problem of energy scarcity in the future WSN applications and Internet-of-Things (IoT) sensors [29]. Also, by employing this WIPT method, a more reliable WSN system with an unwavering quality-of-service (QoS) experience in the context of high throughput, and stable energy supply, can be realized [29-31]. Unfortunately, existing systems based on WIPT methods are faced by an unfairness issue in $\mathrm{EH}$ caused by a problem referred to as doubly-near-far. This situation is a fundamental problem in WPSN systems and affects the information transmission rate of the system [32]. The doubly-near-far situation in WPSN systems initiate unfairness in the energy received by individual sensor nodes in a WPSN system, as the sensor nodes in a network receives varying amount of energy based on a key factor, namely the distance from an IPS. Similarly, the unfairness issue among network sensor nodes is exacerbated by the distance to a base station (BS), where sensor nodes near the BS spend less energy to transfer their separate signals, while the farther sensor nodes consume more energy to transfer their signals. Consequently, some of sensor nodes that are not privileged to be nearer to the BS may not have adequate energy to transfer their separate signals due to energy inadequacy. Thus, based on the unfairness problem in $\mathrm{EH}$ among the sensors in a network, the overall throughput rate of a system is impacted negatively. This similarly translates to the inability of some of the sensor nodes in participating in transferring their vital signals in their allocated time. To enhance fairness among the sensors during $\mathrm{EH}$, as well as to enhance the system throughput, the energy obtained by the individual sensor nodes in the network, including their information transmission rate, can be optimized in a joint manner. Correspondingly, these improvements will circumvent the energy scarcity issue, since throughput QoS is improved with less power.

\section{Related literature}

Currently, the recent works on WPSN solutions considered EH from a single IPS for sensor nodes powering, while only few have exploited the utilization of multiple IPS. In [32], for example, an investigation was carried out on a WPSN application powered by a single IPS. The IPS was employed to wirelessly recharge the batteries of the network sensor nodes. Also, they considered the optimization of the harvesting of energy and the transmission of information timing schedules, to address the effect of unfairness on information transmission rates. Due to a single IPS solution considered in the work, the sensor nodes in the network suffers from a doubly-far-near problem as the sensor nodes that are nearer to the deployed IPS receives more energy compared to the sensors that are far away. Consequently, unfair information transmission rates are experienced by the sensors when this problem is encountered in the network. To improve fairness in information transmission rates among the network sensor nodes, a common-throughput technique was explored and exploited. However, the common-throughput technique is complex and is not a reflection of a practical scenario. The unfairness issues in the energy received and the information transmission rate among the network sensor nodes are addressed in this paper by exploring a heterogeneous multi-class and multiple resource wireless transmission system.

Likewise, in [33], a single IPS-based WPSN system is considered to study the trade-off in the throughput of communication channels. To achieve this, two modes of communication that include half-duplex and full-duplex are employed, and consequently the network devices may operate in any of the modes adopted. To study trade-off in throughput in the downlink and uplink regions, two receiver architectures, namely power-splitting and time switching, were integrated to the network sensor node, while new communication protocols were proposed based on the combination of the communication mode and the receiver architecture. However, the proposed WPSN system suffers from the inherent interference problem that faces full-duplex systems.

An exploration is performed in [34] of the concept of self-recharging of sensor nodes in a network system powered by an IPS. The study exploited a full-duplex mode of communication in a co-located energy transmitter and information receiver architecture that transmits/receives over an in-band frequency such that the energy transmitter as well as the information receiver carries out their communications over a singlefrequency band in a simultaneous fashion. The employed communication mode may be advantageous in improving the spectral efficiency of the operating frequency; however, it suffers from interference problems as the energy transmitter that co-locates with the information receiver causes a complex co-channel interference issue. Since full-duplex communication allows simultaneous communications, the sensor nodes in the full-duplex system in [34] performs data transmission and energy reception concurrently. The network sensor nodes also perform self-recharging as they transfer their independent information. These processes make the system complex as the network sensor nodes are faced with a strong self-interference that causes corruption of data transmissions. Interference cancelation techniques are 
employed; however, it is difficult to achieve perfect cancelation of self-interference in a wireless channel. The interference issues in turn affect the system's overall performance in terms of energy efficiency and achievable throughput rate. The complexity associated to the work in [34] is circumvented in this paper by exploring a half-duplex communication mode in both co-located and separated energy transmitter and information receiver architectures operating in separate time-slots over a single-frequency band. The employed technique in this paper is cost-effective and alleviates the problem of interference faced by the full-duplex approach operating in in-band. The application of both co-located and separate energy transmitter and information receiver architectures employed in this paper tackles the practical doubly-near-far issue faced by the co-located architecture adopted by [34].

A WPSN system for healthcare application is investigated in [35]. The healthcare application was powered by an IPS, and new strategies are proposed for the optimization of the sensor signal transfer rate to the network access point. The study explored two cases that include abnormal and normal situations for transmissions under two schemes, namely time switching and power splitting. For example, the exploration of the abnormal transmission was investigated at the network sensor node under the time switching scheme, while a normal transmission was studied at the network sensor node using the power splitting scheme.

The utilization of multiple IPS is investigated in [36], which considered the optimization of the harvesting of energy and the transmission of information timing schedules of a WPSN system for an on-body application in a dynamic environment. The proportion of the time period earmarked to harvesting for an individual IPS to recharge a sensor node was calculated, which is a function of the movement of the object carrying the sensor nodes. This work did not consider network heterogeneity, as different network specifications are key for a practical system and optimal utilization of resources. The limitation is catered for in this paper to develop a more practical system.

In [37], the optimization of an EH-based WSN system powered by an IPS is considered. The essence of the work is to investigate the maximization of the sum-throughput of the sensors contained in the network with respect to their individual information communication throughput requirement. However, this work is limited by the available energy resource and consequently undermines the potential of the network sensor nodes in meeting their required information transfer rate due to the inherent hardware doubly-near-far condition in wireless-powered WSN systems. This phenomenon is addressed in this work through the deployment of multiple energy resources, which potentially power the network sensor nodes, regardless of their distances, to meet their required information transmission rates.

In [38], the optimization of a WPSN system information transmission and energy harvesting timing schedules was investigated in a joint fashion. The deployment of the sensors in the network was predetermined to target some strategic positions, allowing the control of distances of the sensor nodes to the available IPS. This facilitates fairness in the context of the distances among the sensor nodes to the available energy resources. As a result, equal optimal energy harvesting timing is earmarked to individual sensor nodes for energy harvesting. This work did not consider random deployment of sensors, giving scope for the consideration of network heterogeneity to cater for a more real-world situation in this paper. Unlike the predetermined deployment scenario in [38], in this work, sensor nodes are classified based on their distance specifications using the heterogeneity concept.

In addition to the efforts made in developing optimized energy-efficient WSN systems, reference [39] considered the development of a distributed approach for the estimation and control of dynamic states for seeking an optimal solution to node coordination problem. Because of the interference issue often experienced in a multi-radio wireless system, which often causes data corruption during data communication and significant energy consumption compared to the case of a single-radio wireless system, the authors in [40] explored and exploited an efficient learning automata algorithm to the problem of channel allocation (or assignment) in a wireless system that utilizes multiple radios to improve the throughput rate, delay, and energy consumption of the network. However, an automata algorithm may experience some possible failures as it alternates between different states. This gives scope for the improvement of the automata-based solution to enhance the efficiency of the network.

In this work, a new WPSN is proposed. For efficient resource allocation, and to also realize a more practical system, network heterogeneity is considered. Heterogeneous WSNs is a class of networks where sensor nodes have different properties in terms of distance specification and resources allocation. More often, WSNs are treated as homogeneous, whereas in real scenarios, the networks may have different properties. Consequently, realistic WSNs may not be achieved in homogeneous sensor networks. Heterogeneity is a key design consideration for the realization of efficient and workable systems that are capable of solving several needs. Therefore, the concept of heterogeneous networks is employed in this work to classify sensor nodes based on their distance specifications and deployment strategy. As 
a consequence, a multi-class network is formed, containing class A and class B networks. Class A network sensor nodes are distributed in a predetermined manner to meet some specific design goals, while the sensors in class B are deployed in a random pattern.

Different from [36], equal optimal EH time is provided to the sensor nodes in class A network because of their nearness to the IPS, while a new parameter $T_{\mathrm{EH}}$ is introduced to allot different energy harvesting periods to class B sensors based on their distance from the IPS. Based on the new harvesting time period parameter, an optimal shorter time is allotted to the class B sensors that are near to the IPS, while an optimal larger time is allotted to the class B sensors that are far from the IPS in the downlink (DL). Moreover, to achieve similar signal communication rates within the sensor nodes in the uplink (UL), the sensors which are considered far from the BS are allocated a longer information transmission time to ensure that they have enough time to transfer their separate signals. To achieve this, a new algorithm is proposed to achieve efficient allocation of optimal harvesting time to individual sensors based on their class of network, in order to enhance the system overall throughput rate.

Most often, the time-multiplexing receiver model is employed in WPSN systems because of its installation simplicity, portability, and suitability for efficient harvesting of energy from RF signals [25, 36, 37]. Unfortunately, the current WPSN solutions which are developed based on time-multiplexing are confronted with a number of issues when there are no efficient strategies in place [36]. Such issues range from unfairness in EH time allocation, interference problems caused by energy transmission in the context of multiple IPS, to unequal information communication rates within the sensors in a network [41, 42]. A multi-class, multiple-intended-source (MCMIS) WPSN system is proposed for monitoring the quality of water in water stations, to address the above-mentioned issues. As well, this paper is intended to ensure that individual sensors in the network are efficient enough to obtain adequate energy for delivering their acquired signals with the desired QoS. The major contributions of this paper are fourfold as highlighted below:

1. A new communication scheme that employs time division multiple access (TDMA) is developed to efficiently solve the wireless energy and information transmission scheduling problem.

2. The optimization of the downlink time and the uplink time for energy and information transmissions are achieved with the new TDMA scheme in a joint fashion, to enhance the system overall throughput rate.
3. Optimal allocation of energy resources to the heterogeneous sensor nodes based on their class of network.

4. A new algorithm is developed to improve fairness in resource allocation between the sensor nodes in different classes of a heterogeneous WSN.

There is no prior work on a MCMIS WPSN system for monitoring water quality that studied this problem, to the best of our knowledge. The structuring of this study is highlighted as follows. Following from the review of related works in literature in Section 2, the structure of a sensor node devoted to monitoring water quality is presented in Section 3. The section expounds the proposed MCMIS WPSN system architecture and the proposed TDMA protocol for the system. The proposed model for the new system wireless channel is described in Section 3. This section also contains the optimization of the energy and information transmissions rate problem, as well as an efficient algorithm for multiple IPS allocation and information transmission timing. The discussion of the proposed WPSN system sum-throughput and fairness results are considered in Section 4, which validate the formulated sum-throughput optimization problem. The conclusion of the paper is contained in Section 5.

The notations and terms used in this paper are contained in Table 1.

\section{Methodology}

\subsection{Sensor node hardware design for monitoring water quality}

This section gives a simple overview of the building blocks of a sensor node for monitoring water quality parameters. The water quality sensors are portable, but powerful tools used for monitoring the microbial and the chemical parameters of water quality at water stations. An integral component of a water quality sensor is the communication technology. Communication technologies can be classified into two categories, namely local communication technology and remote communication technology. The local communication technology is used to connect a sensor to another sensor, as well as a BS. The remote communication technology is responsible for delivering water quality information to a remote center. The remote communication technology acts as an internet gateway in the network. An internet gateway simply means an internet access point via which the system is connected to the internet.

The water quality sensors are made up of four essential modules, namely sensor, micro-controller, power supply, and communication. The sensor module is used for measuring the desired parameter of water quality in the form of analog information, and converting the 
Table 1 List of abbreviations

\begin{tabular}{|c|c|c|c|}
\hline $\mathrm{ADC}$ & Analog-to-digital converter & $m_{c, a i} g_{c, b}$ & $\begin{array}{l}\text { Class A channel power gain for UL channels; } \\
\text { class B channel power gain for UL channels }\end{array}$ \\
\hline $\mathrm{BS} / \mathrm{C}_{1}$ & Base station & $n_{c, a} ; u_{c, b}$ & $\begin{array}{l}\text { Class A channel power gain for DL channels; } \\
\text { class B channel power gain for DL channels }\end{array}$ \\
\hline CSI & Channel state information & \ulcorner & SNR gap \\
\hline $\mathrm{DL}$ & Downlink & $\sigma^{2}$ & Noise power \\
\hline $\mathrm{EH}$ & Energy harvesting & $j_{c} ; \xi_{0}$ & $\begin{array}{l}\text { Time-length of class A energy harvesting; time-length } \\
\text { of class B energy harvesting }\end{array}$ \\
\hline IPS & Intended RF power sources & $\zeta_{a i} \xi_{b}$ & $\begin{array}{l}\text { Time-length of class A information transmission; } \\
\text { time-length of class B information transmission }\end{array}$ \\
\hline loT & Internet-of-Things & $x_{c, a i} x_{c, b}$ & $\begin{array}{l}\text { Power signal received by senor } a \text {; Power signal } \\
\text { received by senor } b\end{array}$ \\
\hline ISM & Industrial, scientific and medical & $z_{a i} z_{b}$ & $\begin{array}{l}\text { Background noise at sensor } a \text {; Background noise } \\
\text { at sensor } b\end{array}$ \\
\hline MCMIS & Multi-class, multiple-intended-source & $E_{a i} E_{b}$ & $\begin{array}{l}\text { Total energy received by sensor } a \text {; total energy } \\
\text { received by sensor } b\end{array}$ \\
\hline OAERA & $\begin{array}{l}\text { Optimization algorithm for efficient } \\
\text { resource allocation }\end{array}$ & $j_{c i} \xi_{0}$ & $\begin{array}{l}\text { Time-length of class A energy harvesting; time-length } \\
\text { of class B energy harvesting }\end{array}$ \\
\hline QoS & Quality-of-service & $\zeta_{a i} \xi_{b}$ & $\begin{array}{l}\text { Time-length of class A information transmission; } \\
\text { time-length of class B information transmission }\end{array}$ \\
\hline RF & Radio frequency & $z_{a} ; z_{b}$ & $\begin{array}{l}\text { Background noise at sensor } a \text {; Background noise at } \\
\text { sensor } b\end{array}$ \\
\hline TDMA & Time division multiple access & $E_{a} ; E_{b}$ & $\begin{array}{l}\text { Total energy received by sensor } a \text {; total energy } \\
\text { received by sensor } b\end{array}$ \\
\hline UL & Uplink & $\varepsilon_{a} ; \varepsilon_{b}$ & RF-to-DC conversion efficiency \\
\hline WIPT & $\begin{array}{l}\text { Wireless energy transmission and } \\
\text { wireless information transmission }\end{array}$ & $P_{a i} P_{b}$ & $\begin{array}{l}\text { Average energy consumed by sensor } a \text { for data } \\
\text { transmission; average energy consumed by sensor } b \\
\text { for data transmission }\end{array}$ \\
\hline WSNs & Wireless sensor network & $\psi_{a i} \psi_{b}$ & $\begin{array}{l}\text { Fixed allowable portion of energy for sensor } a \text { data } \\
\text { transmission; fixed allowable portion of energy for } \\
\text { sensor } b \text { data transmission }\end{array}$ \\
\hline WPSN & Wireless powered sensor network & $X_{C_{1}, a} ; X_{c_{1}, b}$ & $\begin{array}{l}\text { Signal received by the BS from sensor } a \text {; signal } \\
\text { received by the BS from sensor } b\end{array}$ \\
\hline$a \in\left\{a_{1}, a_{2}, . ., A\right\} ; b \in\left\{b_{1}, b_{2}, . ., B\right\}$ & $\begin{array}{l}\text { Set of sensor nodes in class A; set of } \\
\text { sensor nodes in class B }\end{array}$ & $x_{a i} x_{b}$ & $\begin{array}{l}\text { Sensor } a \text { arbitrary random signal; sensor } b \text { arbitrary } \\
\text { random signal }\end{array}$ \\
\hline$c \in\left\{c_{1}, c_{2}, \ldots, C\right\}$ & Set of intended RF power sources & $\varepsilon_{a} ; \varepsilon_{b}$ & RF-to-DC conversion efficiency \\
\hline$\tilde{m}_{c, a} ; \tilde{g}_{c, b}$ & $\begin{array}{l}\text { Complex variable of class A UL channels } \\
\text { from sensor } a \text { to the BS; complex variable } \\
\text { of class B UL channels from sensor } b \text { to } \\
\text { the BS }\end{array}$ & $P_{a i} P_{b}$ & $\begin{array}{l}\text { Average energy consumed by sensor } a \text { for data } \\
\text { transmission; average energy consumed by sensor } b \\
\text { for data transmission }\end{array}$ \\
\hline$\tilde{n}_{c, a ;} ; \tilde{u}_{c, b}$ & $\begin{array}{l}\text { Complex variable of class A DL channels } \\
\text { from an IPS } c \text { to sensors } a \text {; complex } \\
\text { variable of class B DL channels from an } \\
\text { IPS } c \text { to sensors } b\end{array}$ & $\psi_{a i} \psi_{b}$ & $\begin{array}{l}\text { Fixed allowable portion of energy for sensor } a \text { data } \\
\text { transmission; fixed allowable portion of energy for } \\
\text { sensor } b \text { data transmission }\end{array}$ \\
\hline$x_{c, a i} x_{c, b}$ & $\begin{array}{l}\text { Power signal received by senor } a \text {; power } \\
\text { signal received by senor } b\end{array}$ & E.coli & Escherichia coli \\
\hline $\mathrm{pH}$ & $\begin{array}{l}\text { Potential of hydrogen (in logarithmic scale } \\
\text { standard) }\end{array}$ & & \\
\hline
\end{tabular}

measured information into a digital form through an analog-to-digital converter (ADC).

The micro-controller module is responsible for the coordination of the processes that integrates the sensor module with other modules in a way to execute instructions that relates to the measurements of the sensor module. Other key functions carried out by the micro-controller involves the collection of the information measured by the sensor unit, storing of the gathered measurements in its storage chip, and transferring of the information collected using the communication technology of the communication module to a BS. 
The communication module is important in the water quality sensor node architecture as it provides a suitable platform for water quality information transmission, and reception of important control signals. The communication module is usually implemented as an RF transceiver. The RF transceiver is equipped with an antenna, and has the capabilities for both information transmission and reception. The CC2420 ZigBee radio is an example of a communication technology for local information transmission, and is defined in the IEEE 802.14.4 specification [43]. The ZigBee radio is considered suitable to be employed in this work because of its low-cost and low-power features. Each of the ZigBee-based water quality sensors communicates directly with a local BS over the license-free ISM bands (such as $2.4 \mathrm{GHz}$ and $915 \mathrm{MHz}$ ). Through a remote communication technology employed at the BS, which acts as a gateway to the internet (such as $2 \mathrm{G}, 3 \mathrm{G}$, or LTE networks), the water quality information received from the sensors is delivered to the remote monitoring stations [2].

The power supply section is a crucial unit in water quality sensor node architecture as it provides energy within the node for powering different modules. The power supply unit may be composed of key devices like an energy harvester and a battery. In this work, an RF-based energy harvester from Powercaster (for example the P2110 device) [44] is considered, and incorporated in the power supply unit for harvesting RF energy from an IPS to recharge the water quality sensor in-built batteries. The RF energy harvester works by converting the RF energy received from an IPS into electrical energy through an RF-to-DC converter. The energy is suitable for powering the sensor node. A typical structure of a WSN system that employs water quality sensors devoted to the monitoring a body of water and its quality is presented in Fig. 1.

\subsection{System architecture design}

In the system architecture, a WPSN system powered by intended RF power sources (IPS) is considered. The system contains two classes of heterogeneous networks. Let the water quality sensors $a$ in class A be denoted by $a \in$ $\left\{a_{1}, a_{2}, . ., A\right\}$, while the water quality sensors $b$ in class $\mathrm{B}$ is denoted by $b \in\left\{b_{1}, b_{2}, . ., B\right\}$. Also, a set of IPS represented by $c \in\left\{c_{1}, c_{2}, . ., C\right\}$ are distributed in the system at specified positions. To provide sufficient energy for powering the water quality sensor nodes, more IPS devices are deployed. The sensor nodes $a$ in class A are distributed in a determined fashion to target some strategic locations, while the sensors in class B are deployed in a random manner, as presented in Fig. 2. The main essence of the two classes of network considered in this study is to cater for several needs, for example the enhancement of effective monitoring of different parameters of water quality such as $\mathrm{pH}$ and $E$. coli.

The multi-class approach employed in this work helps to properly classify the sensor nodes based on their distance specifications and deployment strategy, as depicted in Fig. 2. The IPSes are employed to achieve wireless transmission of energy to the sensors

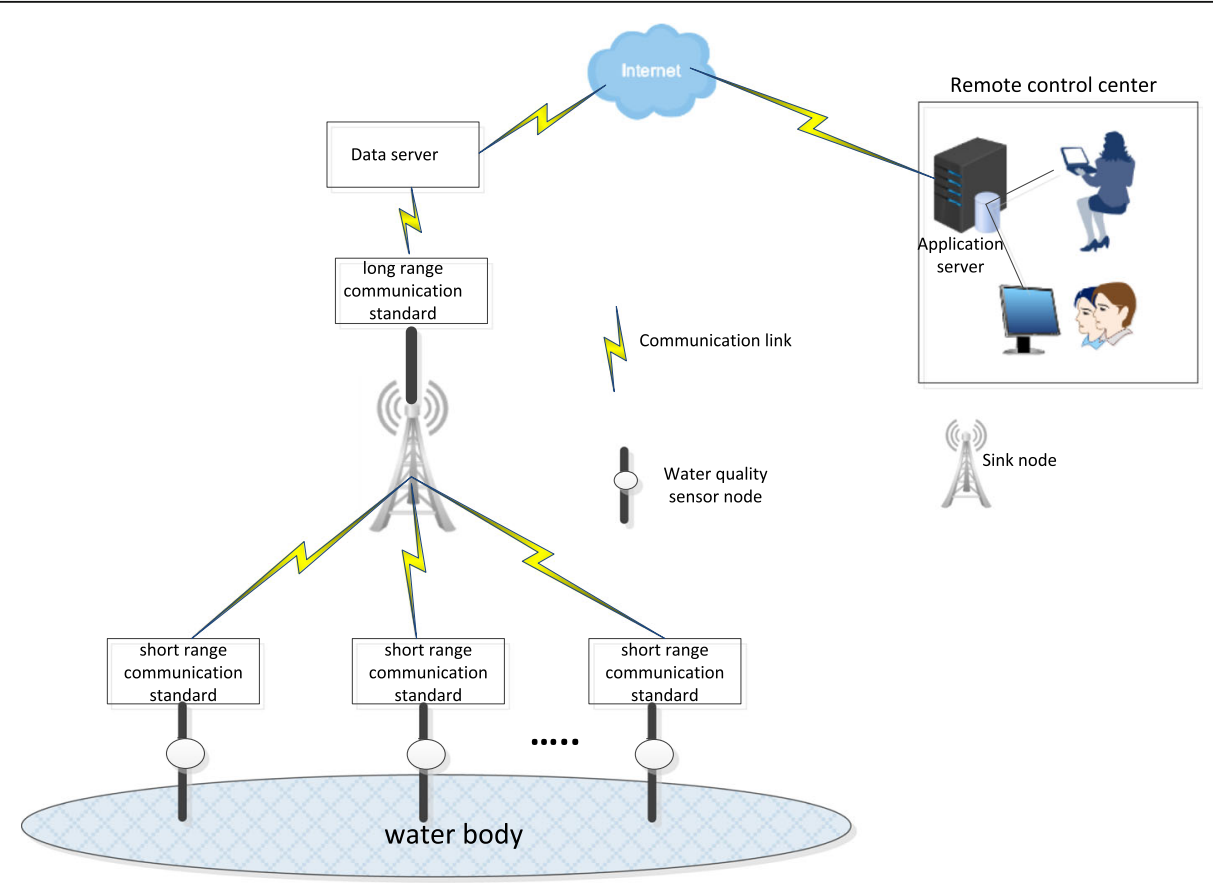

Fig. 1 A typical water quality monitoring system model 


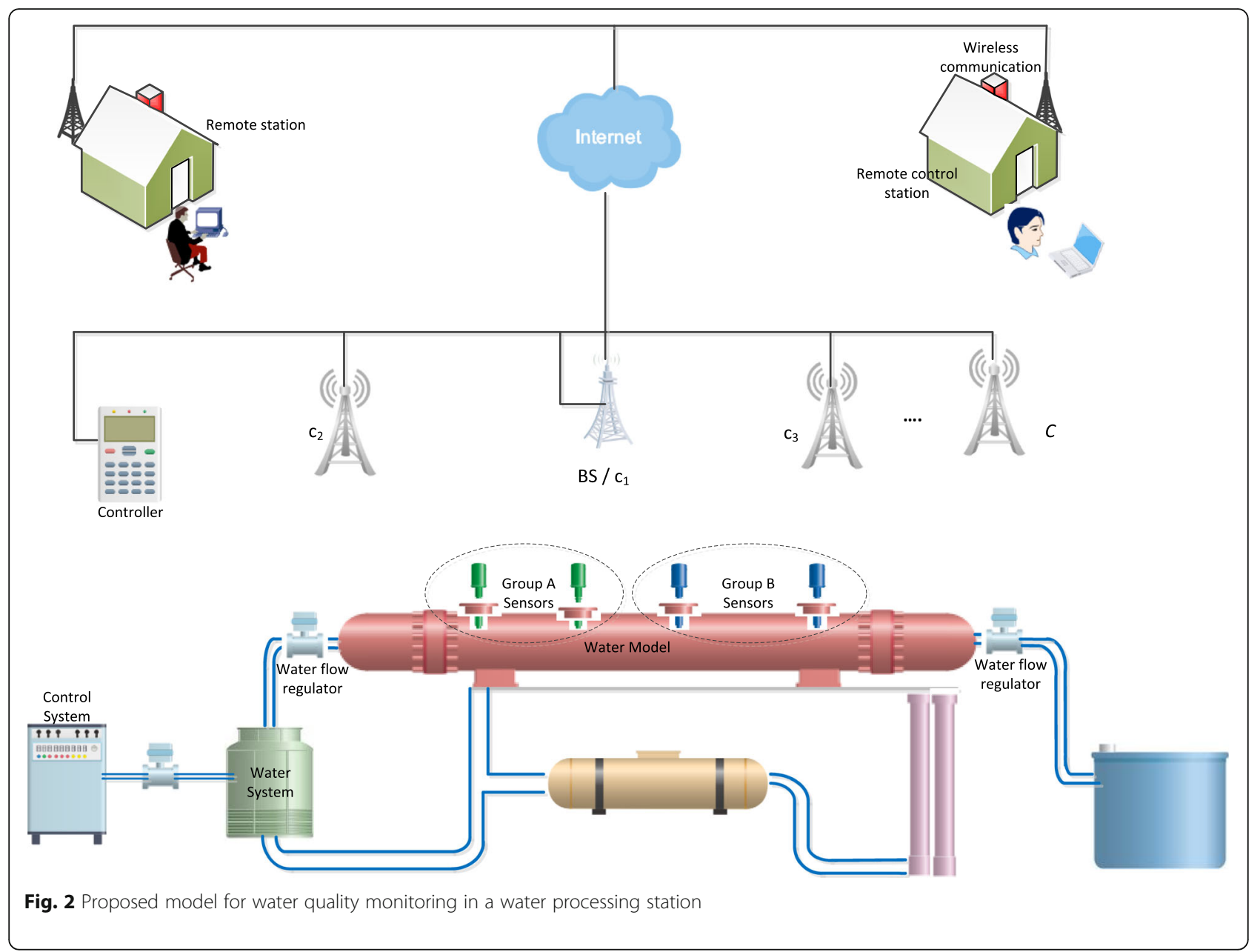

contained in the two classes of network during the DL phase, while only $c_{1}$ has the capability for both wireless energy transmission and wireless information reception in the DL and UL phases. In addition, it is equipped with an internet access capability for remote delivery of water quality information to water control centers. A controller is employed to connect the IPSes, controlling their operation based on the newly proposed TDMA protocol, which circumvents any occurrence of interference in energy transmissions. The new TDMA protocol is given in Fig. 3 . The controller switches the available IPSes on and off at a calculated time, in a sequential manner. To create a suitable platform for the sensors deployment, a section for monitoring the quality of water, which allows constant water flow, is designed as in $[2,45,46]$. The water body that is scheduled for monitoring is pumped to the designed water section in an enclosed location.

In the system architecture, the sensor nodes $a$ in class $\mathrm{A}$ are provided with equal optimal EH time, because of their nearness to the IPS. Unlike the sensor nodes in class A, there are different distances within the sensor nodes in class $\mathrm{B}$ because of the random approach employed for their deployment. Therefore, there may be some significant variations in the energy a sensor node in class $B$ is able to harvest in a DL-EH block. This situation is an inherent issue in WPSNs that is typically referred to as the doubly-near-far problem. When this problem is encountered in a network, the energy that a particular sensor node which is not far from a BS is able to harvest is significant compared to the energy that another sensor node which is far from the BS is able to harvest. This can be attributed to the condition of the wireless channels. To tackle the doubly-near-far issue in this paper, unlike the same optimal EH time that is allotted to class A sensors, different optimal EH time is provided to the individual sensors in class B. In addition, in the UL stage, an optimal information communication period is provided to class $\mathrm{A}$ sensors, as well as class B sensors, based on their distances to the BS, to ensure completeness in the transmission of their individual information to the BS. To achieve this, in 


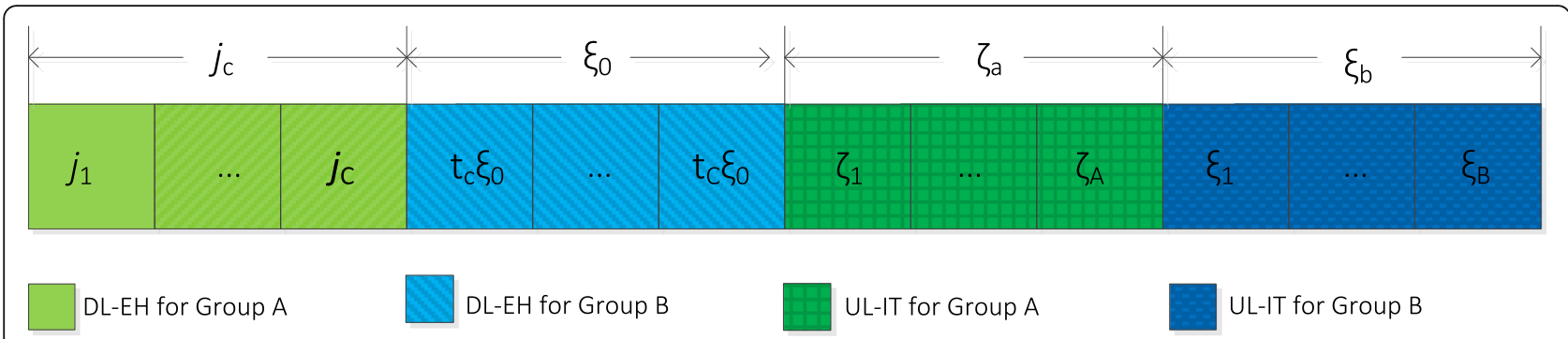

Fig. 3 Proposed TDMA scheme

each information transfer block, the distances to the BS of the sensor nodes $a$ and $b$ in classes A and B, respectively, are considered, and based on this distance, an optimal time is allotted to an individual sensor to transfer its individual information. The new TDMA protocol described here is summarized in Fig. 3.

\subsection{Wireless channel model}

The environment of the application is assumed to be a static environment. As a result, the wireless channels between the sensor nodes and the IPS $c$ are modeled using a quasi-static fading model. The channels that connect the sensor nodes $a$ and $b$ to the BS are denoted with complex variables $\tilde{m}_{c, a}$ and $\tilde{g}_{c, b}$ in the UL phase, for classes A and $\mathrm{B}$, respectively. While the reversed channels that go from an IPS to the sensor nodes $a$ and $b$ are denoted with $\tilde{n}_{c, a}$

and $\tilde{u}_{c, b}$ in the DL phase, for classes A and B. Consequently, the channel power gains for the two classes are derived as $m_{c, a}=\left|\tilde{m}_{c, a}\right|^{2}$ and $n_{c, a}=\left|\tilde{n}_{c, a}\right|^{2}$ for class A, and $g_{c, b}=\left|\tilde{g}_{c, b}\right|^{2}$ and $u_{c, b}=\left|\tilde{u}_{c, b}\right|^{2}$ for class B.

In addition, each IPS is assumed to have knowledge of the channel state information (CSI), and as a result employs the CSI knowledge to ensure the transmission of optimal energy to individual sensors in the two classes in an adaptive fashion.

The proposed MCMIS WPSN system is further described as follows:

\subsubsection{Class $A$}

In a particular $j_{\mathrm{c}}$ period, with the application of the TDMA protocol in Fig. 3, an EH time of $0 \leq j_{c} \leq 1, j_{c} \geq 0$, $c=1, \ldots, C$ is allotted to an IPS $c$ to send energy via the DL channels to sensors $a$, while the scheduled time for sensor nodes $a$ for transferring their signals over channel $m_{1}$, a to $\mathrm{BS} / c_{1}$ in the UL phase is represented with time period $\zeta_{a}, a=1,2, \ldots, \mathrm{A}$, with a length of $0 \leq \zeta_{a} \leq 1$. Therefore, the time allocated to an IPS $c$ for energy transmission to the sensor nodes in class $\mathrm{A}$, and the scheduled time for the sensor nodes to communicate their separate signals in the UL phase is given in (1) as follows:

$$
\sum_{\mathrm{c}=1}^{\mathrm{C}} \mathrm{j}_{\mathrm{c}}+\sum_{\mathrm{a}=1}^{\mathrm{A}} \zeta_{\mathrm{a}} \leq 1
$$

In (2), the amount of power that a sensor node receives from an IPS is formulated as follows:

$$
x_{c, a}=\sqrt{n_{c, a}} x_{c}+z_{a}, \forall a=1,2, \ldots, A
$$

where $x_{c, a}$ means the power signal received by senor $a$, and $z_{a}$ indicates the background noise at $a$ as a result of the energy received from an IPS $c . x_{c}$ denotes the arbitrary complex random signal of an IPS $c$ that satisfies $E\left[\left|x_{c}\right|^{2}\right]=P_{c}$, where $P_{c}$ means the IPS $c$ transmission power, and is assumed large enough that the background noise at $a$ is insignificant as a consequence.

In the DL phase, the energy a sensor node $a$ harvests from an IPS $c$, in a given time-slot, is formulated in (3) as follows:

$$
E_{c, a}=\varepsilon_{a} P_{c} n_{c, a} j_{c}, \forall c=1,2, \ldots, C, \forall a=1,2, . ., A
$$

Moreover, the overall energy received by sensor node $a$ from the IPS $c$ is modeled in (4) as follows:

$$
E_{a}=\varepsilon_{a} \sum_{c=1}^{C} P_{c} n_{c, a} j_{c}, \forall a=1,2, \ldots, A
$$

where $\varepsilon_{a}$ denotes the efficiency of the RF-to-DC converter module of sensor node $a$ and is defined as $0 \leq \varepsilon_{a} \leq$ 1 , for $\mathrm{a}=1,2, \ldots, A$. The assumption is made that $\varepsilon_{1}=$ $\ldots=\varepsilon_{A}=\varepsilon$, for simplicity sake.

To optimize the energy consumption of each sensor node $a$, only a fraction of the energy obtained by each of them in (4) is allowed to be consumed for information transmission. Consequently, an average transmission power is defined for the sensor nodes as modeled in (5) as follows:

$$
P_{a}=\frac{\Psi_{a} E_{a}}{\zeta_{a}}, \forall a=1, \ldots, A
$$

In (5), $P_{a}$ is the average transmission power defined for a sensor node $a$., while $\Psi_{a}$ indicates the fixed value 
allowable part of the energy available to $a$ to transfer information to the BS. $\Psi_{a}$ is defined as $\Psi_{a}=\ldots=\Psi_{A}=\Psi$, for convenience. It is important to mention that $1-\Psi$, which is the remaining fraction of the harvested energy, is utilized for operating the internal modules of a sensor node $a$.

The received signal at the BS $c_{1}$ from individual sensors $a$ in each UL time-slot is given by:

$$
x_{c_{1}, a}=\sqrt{m_{1, a}} x_{a}+\mathrm{z}_{c_{1}}, \forall a=1, \ldots, A
$$

where $x_{c_{1}, a}$ means the signal received by the BS $c_{1}$, $x_{a}$ denotes an arbitrary random signal of a sensor node $a$ that satisfies $E\left[\left|x_{a}\right|^{2}\right]=P_{a}$, and $z_{c_{1}}$ is used to denote the background noise at $c_{1}$ as a result of the signal received from a sensor node $a$. For the transmission of information in the UL by sensor $a$ to $c_{1}$, the capacity of the channel is defined as (7), based on Shannon's law [47]:

$$
D_{a}=\zeta_{a} \log _{2}\left(1+\frac{P_{a} m_{1, a}}{\Gamma \sigma^{2}}\right)
$$

In (7), the signal transfer time (related to the channel bandwidth of the system) is denoted with $\zeta_{a}$, the SNR gap is represented with $\Gamma$, and the noise power is represented with $\sigma^{2}$. The maximum throughput that sensor $a$ can achieve in $\mathrm{b} / \mathrm{s} / \mathrm{Hz}$ is represented with $R_{a}$ and is defined in (8) as follows:

$$
R_{a} \leq \zeta_{a} \log _{2}\left(1+\frac{P_{a} m_{1, a}}{\Gamma \sigma^{2}}\right)
$$

By substituting (5) and (4) into (8), the throughput rate can be derived in the form of

$$
R_{a}(j, \zeta)=\zeta_{a} \log _{2}\left(1+\alpha_{a} \frac{\sum_{c=1}^{C} j_{c}}{\zeta_{a}}\right), \forall a=1,2, \ldots, A
$$

where $j=\left[j_{1}, j_{2}, j_{3}, \ldots, j_{C}\right], \zeta=\left[\zeta_{0}, \zeta_{1}, \ldots, \zeta_{a}\right]$, and $\alpha_{a}$ represents the SNR at $c_{1}$ and is defined in (10) as follows:

$$
\alpha_{a}=\frac{\Psi_{a} \varepsilon_{a} m_{1, a} \sum_{c=1}^{C} P_{c} n_{c, a} j_{c}}{\Gamma \sigma^{2}}, \forall a=1, ., A
$$

Consequently, for all the of sensors $a$, the sum-throughput is defined in (11) as follows:

$$
R_{\text {sum }}(j, \zeta)=\sum_{a=1}^{A} R_{a}(j, \zeta), \forall a=1,2, . ., A
$$

\subsubsection{Class $B$}

In class $B$, an optimal $E H$ time with a length of $0 \leq$ $t_{1} \xi_{0} \leq 1$ is calculated and allotted to an IPS $c$ to transmit energy to each individual sensor $b$ over the DL communication channels, while an optimal period of time $\xi_{b}$ is apportioned to a sensor $b$ to communicate its signal through the UL links to $c_{1}$ over a channel $g_{1, b}$. The apportioned time $\xi_{b}, b=1,2, \ldots, B$, has a length of $0 \leq \xi_{b} \leq$ 1. Therefore, the time period apportioned to an IPS $c$ for the transmission of energy, as well as the time period apportioned to sensor nodes $b$ for communicating their different signals to the BS, is formulated in (12) as follows:

$$
\sum_{c=1}^{C} t_{c} \xi_{0}+\sum_{b=1}^{B} \xi_{b} \leq 1
$$

The amount of power that a sensor node receives from an IPS is formulated as follows:

$$
x_{c, b}=\sqrt{u_{c, b}} x_{c}+z_{b}, \forall b=1,2, \ldots, B
$$

In the DL phase, the energy a sensor node $b$ harvests from an IPS $c$, in a given time-slot, is formulated in (14) as follows:

$$
E_{c, b}=\varepsilon_{b} P_{c} u_{c, b} t_{c} \xi_{0}, \forall c=1,2, \ldots, C, \forall b=1,2, . ., B
$$

The total energy received by sensor node $b$ from the IPS $c$ is modeled in (15) as follows:

$$
E_{b}=\varepsilon_{b} \sum_{c=1}^{C} P_{c} u_{c, b} t_{c} \xi_{0}, \forall b=1,2, \ldots, B
$$

Once again, it is assumed for convenience that $\varepsilon_{1}=\ldots$ $=\varepsilon_{B}=\varepsilon$.

From (15), a part of the energy obtained by each sensor $b$ is consumed for information communication in the UL phase and is formulated in (16) as follows:

$$
P_{b}=\frac{\Psi_{b} E_{b}}{\xi_{b}}, \forall b=1,2, \ldots, B
$$

where $P_{b}$ is the average transmission power defined for a sensor node $b$, while $\Psi_{b}$ indicates the allowable part of the energy contained in $b$ for information communication to the BS, which is fixed. $\Psi_{b}$ is defined as $\Psi_{b}=\ldots=\Psi_{B}=\Psi$, for convenience. The rest of $1-\Psi$ is utilized for operating the modules of a sensor node $b$.

The received signal at $c_{1}$ from individual sensors $b$ in each UL time block is:

$$
x_{c_{1}, b}=\sqrt{g_{1, b}} x_{b}+z_{c_{1}}, b=1, \ldots, B
$$

The attainable throughput rate in $\mathrm{b} / \mathrm{s} / \mathrm{Hz}$ of sensor node $b$ is defined as follows: 


$$
R_{b}(t, \xi)=\xi_{b} \log _{2}\left(1+\gamma_{b} \frac{\sum_{c=1}^{C} t_{c} \xi_{0}}{\xi_{b}}\right), \forall b=1,2, . ., B
$$

where $t=\left[t_{1}, t_{2}, t_{3}, \ldots, t_{C}\right], \xi=\left[\xi_{0}, \xi_{1}, \ldots, \xi_{b}\right] . \gamma_{b}$ is the SNR received at $c_{1}$, which is caused by the transferred information from sensor node $b$. It is defined in (19) as follows:

$$
\gamma_{b}=\frac{\Psi_{b} \varepsilon_{b} g_{1, b} \sum_{c=1}^{C} P_{c} u_{c, b} t_{c}}{\Gamma \sigma^{2}}, \forall b=1, . ., B
$$

Hence, for all of the sensors $b$, the sum-throughput is defined in (20) as follows:

$$
R_{\text {sum }}(t, \xi)=\sum_{b=1}^{B} R_{b}(t, \xi), \forall b=1,2, . ., B
$$

\subsection{Maximization of attainable throughput}

The maximization of the WPSN system attainable throughput is described in this segment. To achieve this, a sum-throughput optimization strategy is employed. Based on the optimization technique, the timing schedules for the harvesting of energy and transmission of information by sensor nodes $a$ and $b$ were optimized in joint fashion. With this, an improved fairness in the allocation of harvesting timing, as well as fairness in the rates of the sensor nodes information transmission, is achieved. Consequently, an enhanced system overall throughput rate is achieved with minimal energy consumption. The general representation of the system attainable throughput is formulated as a maximization problem in (P1). From (1), we have:

(P1):

$$
\max _{j, \zeta, t, \xi} R_{\text {sum }}(j, \zeta)+R_{\text {sum }}(t, \xi)+\ldots+R_{\text {sum }}(s, v)
$$

subject to:

$$
\begin{aligned}
& \sum_{c=1}^{C} j_{c}+\sum_{c=1}^{C} t_{c} \xi_{0}+\sum_{a=1}^{A} \zeta_{a}+\sum_{b=1}^{B} \xi_{b} \leq 1 \\
& j_{c} \geq 0, \forall c=1,2, . ., C \\
& t_{c} \geq 0, \forall c=1,2, . ., C \\
& \zeta_{a} \geq 0, \forall a=1,2, . ., A \\
& \xi_{b} \geq 0, \forall b=1,2, . ., B
\end{aligned}
$$

The objective function of the optimization problem is given in (21), while the constraints of the optimization problem are (21a) to (21e). Constraint (21a) is the timing schedules for energy harvesting and information transmission. The non-negative constraints (21b), (21c), (21d), and (21e) are defined for the decision variables, while variables $j, \mathrm{t}, \zeta, \xi$ are unknown in (P1). The maximization problem in (P1) is a non-convex problem since (9) and (18) contain a $\log$ function. By exploiting the structure of the problem, variable $t_{c} \xi_{0}$ is changed to $\xi_{0, c}$, and the natural $\log$ form of the $\log$ function is obtained. They are substituted in (9) and (18) respectively. Based on this development, the optimization problem in (P1) is transformed to a convex problem. The newly generated problem from the original problem is defined as (P2). The proof for the new problem is provided in Appendix 1. Consequently, the newly transformed problem is solvable by employing any standard convex approach $[2,48]$.

Moreover, in order to provide a solution to unfairness in energy harvesting as a result of the transformation, we formulated a new problem as (P3) to guarantee the optimality of $j$ and $t$, which is indicated as $j^{*}$ and $t^{*}$. Consequently, these values $j^{*}$ and $\left.\mathrm{t}^{*}\right)$ are employed in (P1). The formulation of the minimization problem for addressing the unfairness in energy harvesting among the sensors is expressed in (22) as follows:

(P3):

$$
\min _{j^{*}, t^{*}} E\left[\left(E_{a}-\bar{E}_{a}\right)^{2}+\left(E_{b}-\bar{E}_{b}\right)^{2}\right]
$$

s.t:

$$
\begin{aligned}
& \sum_{c=1}^{C} j_{c}+\sum_{c=1}^{C} t_{c}=1 \\
& j_{c} \geq 0, \forall c=1,2, . ., C \\
& t_{c} \geq 0, \forall c=1,2, . ., \mathrm{C}
\end{aligned}
$$

In (22), the minimum energy received by $a$ and $b$ is defined by $\bar{E}_{a}$ and $\bar{E}_{b}$, and is calculated based on (23) and (24).

$$
\begin{aligned}
& E_{a}=E\left(E_{a}\right)=\frac{\sum_{a=1}^{A} E_{a}}{A} \\
& E_{b}=E\left(E_{b}\right)=\frac{\sum_{b=1}^{B} E_{b}}{B}
\end{aligned}
$$

(P2) is contingent to variables $j, \mathrm{t}, \varepsilon_{0}$, which are unknown. To determine the intermediate harvested energy for $E_{a}, a=1,2 \ldots, A$, as well as $E_{b}, b=1,2 \ldots$, $B$, arbitrary values could be used for $j_{c}$ and $\xi_{0}$. The proof for determining optimal $j^{*}$ and $t^{*}$ is provided in Appendix 2.

In addition, to handle multiple IPS allocation in an efficient manner to ensure fairness in harvesting and signal transmission rates among class $\mathrm{A}$ and class $\mathrm{B}$ 
sensors, an efficient algorithm is developed. Moreover, to determine the rates of fairness in resource allocation and signal transmission in the system, the concept of Jain's fairness index [38, 49] is employed, as expressed in (25).

$$
J F=\frac{\left(\sum_{k=1}^{v} R_{\nu}(\beta)\right)^{2}}{v \cdot \sum_{k=1}^{v}\left(R_{\nu}(\beta)\right)^{2}}
$$

In (25), $v=a+b$, which represents the complete network of sensors in classes A and B. $\beta=(j+\zeta)+(\xi)$ is the combined time length for classes $A$ and $B$ sensor nodes. While, the overall aggregate of the sum-throughput of class A and class B is defined by $R_{v}(\beta)=R_{a}(j, \zeta)+R_{b}(\xi)$. For the sake of performance measurement, the best case, as well as the worst case, of the overall sensor nodes in class A and class B, is expressed by (26) as follows:

$$
\frac{1}{V} \leq J F \leq 1
$$

According to (26), 1 indicates a maximum fairness ratio, while $\frac{1}{V}$ means a minimum fairness ratio.

\subsection{Efficient allocation algorithm for energy and information transmission scheduling}

In this section, an efficient resource allocation algorithm is presented and is defined as Algorithm 1. The essence of the proposed algorithm is to ensure fairness in EH-DL timing schedules among the system sensor nodes. In addition, it is aimed to achieve an enhanced rate of information transfer among the network sensor nodes in the UL. To achieve this, the proposed algorithm optimizes the energy and information transfer timing schedules in a joint fashion, according to the mathematical models presented in Section 3 such that optimal time periods are allocated for both $\mathrm{EH}$ and information transmission to classes $\mathrm{A}$ and $\mathrm{B}$ in the network. As a consequence, Algorithm 1 optimally allots an IPS $c$ to individual sensor nodes at a calculated optimal time period. In a similar vein, to make sure that the sensors in the network are provided with sufficient time for communications in the UL phase, an optimal information transmission time period is calculated and allotted. The implementation of the algorithm is done on the system controller to achieve the optimal control of the switching of the IPS and optimally allocating them to the sensors for enhancing the attainable throughput of the WPSN system.
Algorithm 1: Optimization algorithm for efficient resource allocation (OAERA)

Require: $\left\{a_{1}, a_{2}, \ldots, \mathrm{A}\right\},\left\{b_{1}, b_{2}, \ldots, \mathrm{B}\right\} \triangleright$ sensor nodes $a$ and $b$; $\left\{c_{1}, c_{2}, \ldots, \mathrm{C}\right\} \triangleright \mathrm{IPS} c$

Ensure: $j_{c}^{*}, t_{c}^{*}, \forall c=1,2 \ldots, C \triangleright$ optimal energy harvesting time

$\zeta_{\mathrm{a}}^{*}, \xi_{\mathrm{b}}^{*}, \forall a=1,2 \ldots, \mathrm{A}, b=1,2 \ldots, \mathrm{B} \triangleright$ optimal

information transfer time.

\section{Downlink timing schedule for energy harvesting}

1. Initialization:

2: Let $c \leftarrow 1: C$ for $a \in\left\{a_{1}, a_{2}, \ldots, A\right\}$

3: switch $c$ to $\mathrm{ON}$ for $0 \leq j_{c}^{*} \leq 1$

4: for $a=1: A$ do

5: $\quad$ check for the closest $c$ and allocate to $a$

6. if $c$ is the closest to $a$ then

7. allocate $c$ to $a$, and energy is transferred for an optimal assigned time period using the energy model in Eqn. (4) and $n_{c, a}=10^{-3} d_{c, a}^{-\omega}$

8. otherwise continue with the search

9. end if

10. end for

11. switch OFF $c$

12: Let $c \leftarrow 1: C$ for $b \in\left\{b_{1}, b_{2}, \ldots, B\right\}$

13: switch $c$ to ON for $0 \leq t_{\mathrm{c}}^{*} \leq 1$

14: for $b=1: B$ do

15: find the distance between $b$ and the available $c$ 's

16: with the calculated distances, allocate optimal (short or large) DL-EH time to $b$ and harvest energy from $c$ using the energy model in Eqn. (15)

17: end for and $u_{c, b}=10^{-3} d_{c, b}^{-\omega}$

18. switch OFF $c$

\section{Uplink timing schedule for information transmission}

19: for $a=1: A$ do

20: $\quad$ switch ON $c_{1}$ for $0 \leq \zeta_{\mathrm{a}}^{*} \leq 1, \forall a=1,2, \ldots, A$

21: find the distance between $a$ and $c_{1}$

22: with the calculated distance, allocate optimal (short or large) UL-IT time to $a$ and the models in Eqns. (8) and (9) are employed for the transmission of sensor $a$ information to the BS $c_{1}$, and Eqn. (5) calculates the average energy consumed by sensor $a$ for transmitting information

23: $\quad$ switch OFF $c$

24: end for

25: for $b=1: B$ do

26: $\quad$ switch ON $c_{1}$ for $0 \leq \xi_{\mathrm{b}}^{*} \leq 1, \forall b=1,2, \ldots, B$

27: $\quad$ find the distance between $b$ and $c_{1}$

28: $\quad$ with the calculated distance, allocate optimal (short or large) UL-IT time to $b$ and the models in Eqns. (18) and (19) are employed for the transmission of sensor $b$ information to the BS $c_{1}$, and Eqn. (16) finds the average energy consumed by sensor $b$ for transmitting information

29: $\quad$ switch OFF $c$

30: end for

end

To analyze the complexity or performance of the OAERA algorithm, two key parameters used for 
characterizing the complexity of an algorithm are employed in this study. The parameters are the time complexity and space complexity. Note that the analysis of the time complexity of an algorithm involves the required time to execute an algorithm of a particular size $n$, while the analysis of the space complexity is concerned with the required system resources (such as memory) to execute an algorithm of a particular size $n$. To achieve the characterization of the complexities of the OAERA algorithm, Big-O $(O)$ notation is applied. The time complexity of the OAERA algorithm is $O(A(C$ $+1)+2 B)$. Consequently, the computational time complexity of the algorithm is linear in sensor nodes in $A$ and $B$, and directly proportional to the IPS $C$. The space complexity of the OAERA algorithm is $O(A+B+C)$, which reveals a linear complexity. These indications show that the OAERA algorithm has efficient complexities in the context of time and space. An algorithm with a linear complexity is better than an algorithm with an exponential complexity as in [36] since the efficiency of $n>2^{n}$. Also note that algorithms with exponential complexities are solvable, but not tractable. As a result, they may explode. An exponential-time complexity consumes more time and space resources compared to algorithms with linear complexities, and polynomial time complexities defined by $n^{q}$ where $q \geq 2$, such as quadratic complexity and cubic complexity. It is important to underline that system resources can efficiently take care of linear-time and polynomial-time algorithms as they are solvable and tractable.

\section{Results and discussion}

This section presents the performance of the proposed optimization algorithm by investigating the effects of number of sensors, path-loss exponent, and transmission power on the system, through simulation experiments. In addition, the system performance is verified in a comparative manner in the context of the number of IPS available in the system, and Jain's fairness ratio. Also, to further substantiate the contributions of this work, two networks at unequal distances to the BS are investigated, to showcase the improvement in network performance. Based on simulation experiments, the computational efficiency of the proposed optimization algorithm is shown, while the simulation settings presented in Table 2 are employed to configure the proposed WPSN system. This work assumes similar network parameters as in a recent reference work [36], for comparison purposes.

The implementation of the algorithm was done on the following proposed WPSN system. Two classes of network are considered at a distance of $3 \mathrm{~m}$ apart. In class A, a distance of $6 \mathrm{~m}$ is considered for the placement of one or two sensor nodes by taking the data in the reference work into consideration. In class $\mathrm{B}$, the water
Table 2 System parameters

\begin{tabular}{ll}
\hline Parameter & Value \\
\hline Carrier bandwidth & $1 \mathrm{MHz}$ \\
SNR & $1.5 \mathrm{~dB}$ \\
Noise power & $-114 \mathrm{dBm}$ \\
IPS transmission power & $3000 \mathrm{~mW}$ \\
Efficiency of RF energy conversion & 0.5 \\
Channel path-loss exponent, $\omega$ & 2.0 \\
Allowable portion of energy for & 0.5 \\
information transfer & \\
MAC layer & IEEE 802.15 .4 \\
Operating frequency & $915 \mathrm{MHz}$ \\
Path-loss channel model & $n_{c, a}=m_{c, a}=10^{-3} d_{c, a}^{-\omega}$ \\
Path-loss channel model & $u_{c, b}=g_{c, b}=10^{-3} d_{c, b}^{-\omega}$ \\
\hline
\end{tabular}

quality sensors are distributed at a random distance of 2.5-4 $\mathrm{m}$ from each other-as typical in monitoring the quality of water. In addition, it is possible to vary the distance among the sensor nodes. Furthermore, it is worth mentioning that during optimization, different strategic positions are considered for the IPSes.

\subsection{Algorithm convergence based on iteration number}

In this experiment to investigate the convergence of the system, both the number of sensors and IPSes in the network are fixed, while the number of iterations is changed during the simulation. Figure 4 depicts the convergence of the proposed OAERA algorithm in the context of attainable system sum-throughput per sensor nodes against iteration number. For this experiment, a system configured with two sensors and a system configured with three sensors were investigated. The two systems are powered by five IPS, which is consistent with the reference work [36]. From Fig. 1, it can be noticed that it takes the proposed OAERA algorithm an average run time of 500 iterations to realize an optimal solution, as the iteration number is observed to enhance the system attainable sum-throughput of the two systems. As a result, it is reasonable to utilize 500 iterations for averaging the performance of the system. Thus, results are obtained based on an average of 500 iterations.

\subsection{Path-loss exponent impact on the system attainable throughput}

In the course of this experiment, the number of network sensor nodes is fixed and the channel path-loss exponent is varied to investigate the impact of path-loss exponent. The experiment is repeated for systems configured with two, three, and four sensors. Each of the systems is powered by five IPS. From the results in Fig. 5, it is noticeable that there is a decrease in the system average 


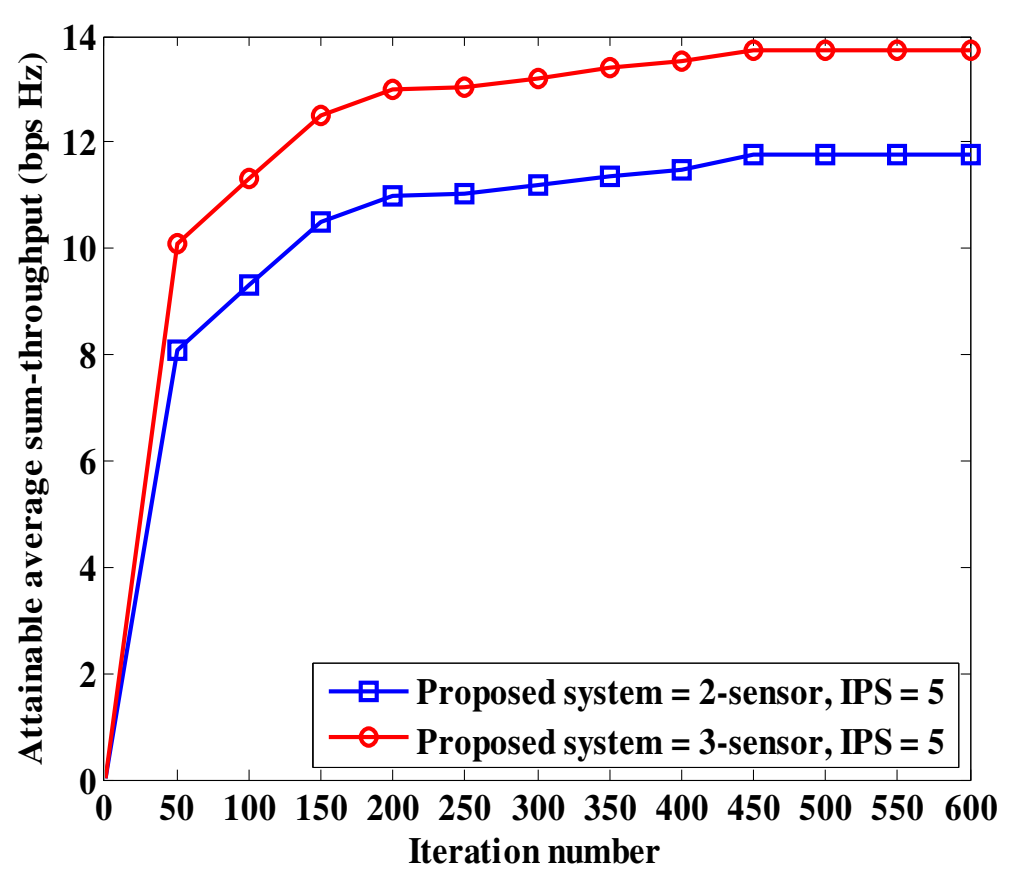

Fig. 4 Attainable average sum-throughput of the system versus iteration number

attainable sum-throughput as the value of path-loss exponent increases. The reduction experienced in the system attainable throughput due to rise in path-loss exponent is valid in the two-sensor, three-sensor, and four-sensor systems. Another observation is that, a system with four-sensor had a higher average attainable sum-throughput compared to the systems with two-sensor and three-sensor. Therefore, it is confirmed that the system performs better when it is configured with a lower path-loss exponent value.

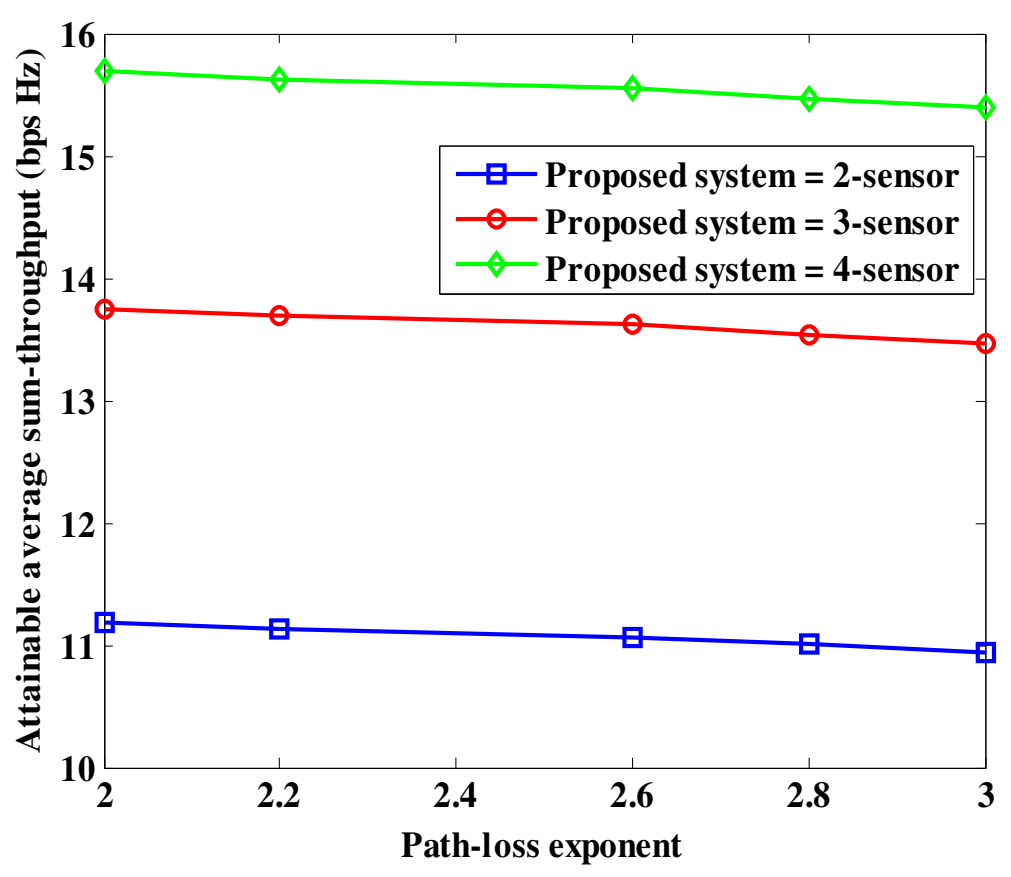

Fig. 5 Attainable average sum-throughput versus path-loss exponent 


\subsection{Performance comparison of systems with different configurations}

In this section, simulation investigations were carried out on the new WPSN application, as well as on an existing WPSN application in [36]. The network sensor nodes are fixed, while the number of IPSes changes in the course of the experiment to investigate the impact of the number of IPSes on the overall attainable throughput rates and fairness index. Based on the simulation experiments, the proposed WPSN system was compared with the existing system in the context of the attainable average sum-throughput, and fairness. As in [36], two different system configurations were considered. One of the systems was configured with two-sensor, while the other was configured with three-sensor. The two systems are powered by five IPS. For the comparison of the proposed system and the existing system, the same simulation software is run, while the algorithm proposed is activated for the WPSN system of this work, and deactivated for the existing WPSN system. Consequently, the proposed WPSN system and the existing WPSN system are compared based on the attainable average sum-throughput and Jain's fairness as shown in Figs. 6 and 7.

From Fig. 6, it is noticeable that the proposed WPSN system outperforms the existing WPSN system, as it achieves an enhanced average sum-throughput. The improvement in the attainable sum-through results is as a result of the newly proposed algorithm. The new optimization algorithm efficiently allocates optimal time to DL-EH and UL-IT. In addition, through the results in Fig. 6, it is easy and straightforward to infer that a two-sensor system that is operated based on the proposed algorithm performs comparably to a three-sensor system that is operated based on the existing algorithm. Moreover, the ratio of fairness in resource allocation between the sensor nodes in the network is investigated by employing the Jain's equation. As a consequence, from Fig. 7, it is observed that the proposed WPSN system achieved enhanced fairness rates when compared to the existing two-sensor system and three-sensor system. This indicates an interesting improvement in fairness in the allocation of resources in the system, thus, addressing the inherent doubly-near-far issue in WPSN systems. From the results, it can be concluded that the proposed optimization algorithm optimizes the system sumthroughput by $26.46 \%$ and $27.18 \%$ for two-sensor and three-sensor, respectively, in comparison to the existing system. Similarly, the ratio of fairness of the proposed system configured with two-sensor and three-sensor indicate improvements of $8.6 \%$ and $8.5 \%$, respectively, compared to the existing system.

\subsection{Comparison based on unequal network distances to the BS}

The effect of unequal network distance to the BS between two classes of network is investigated in this section to emphasize the contributions of the newly

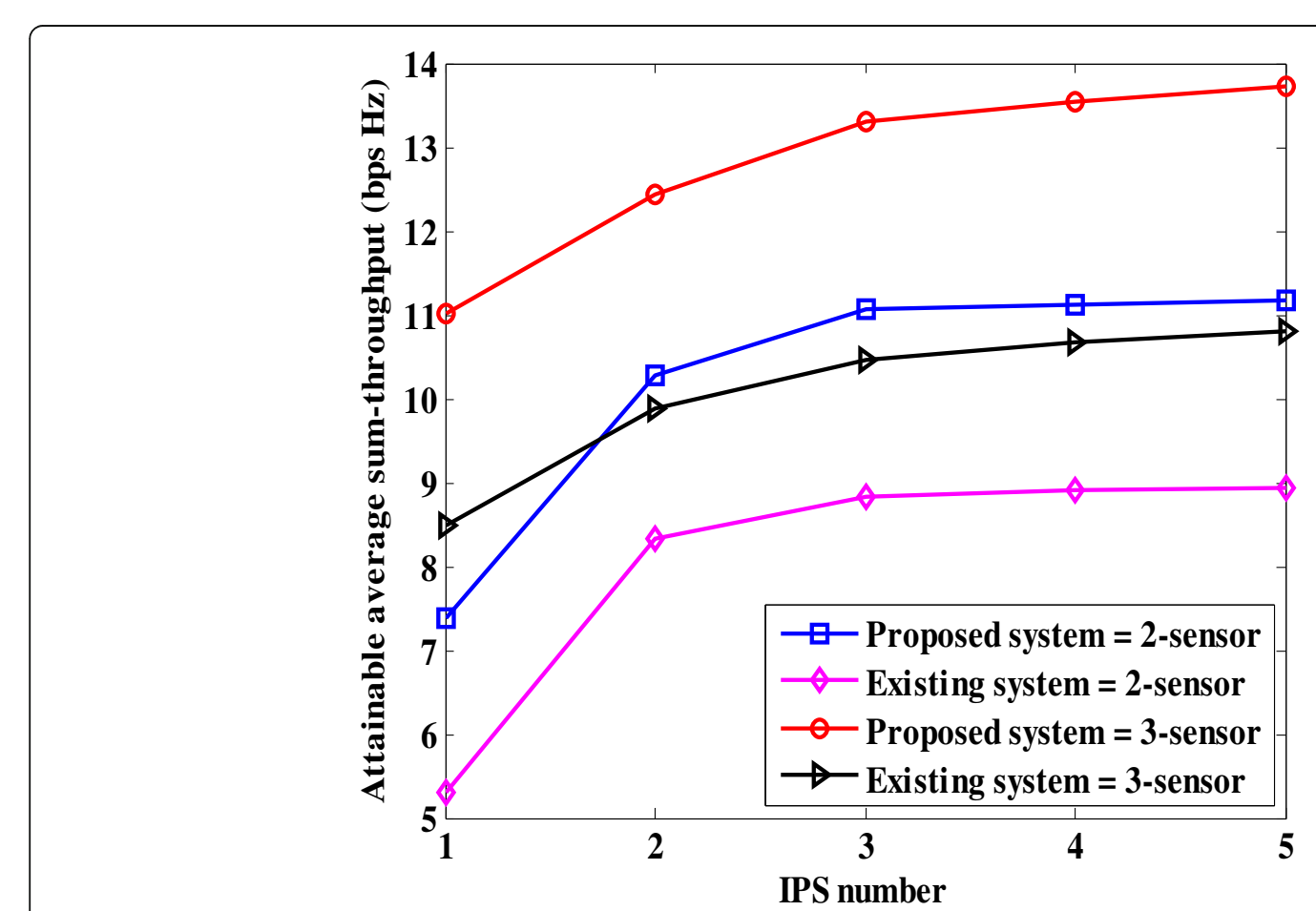

Fig. 6 Attainable average sum-throughput versus number of IPS 


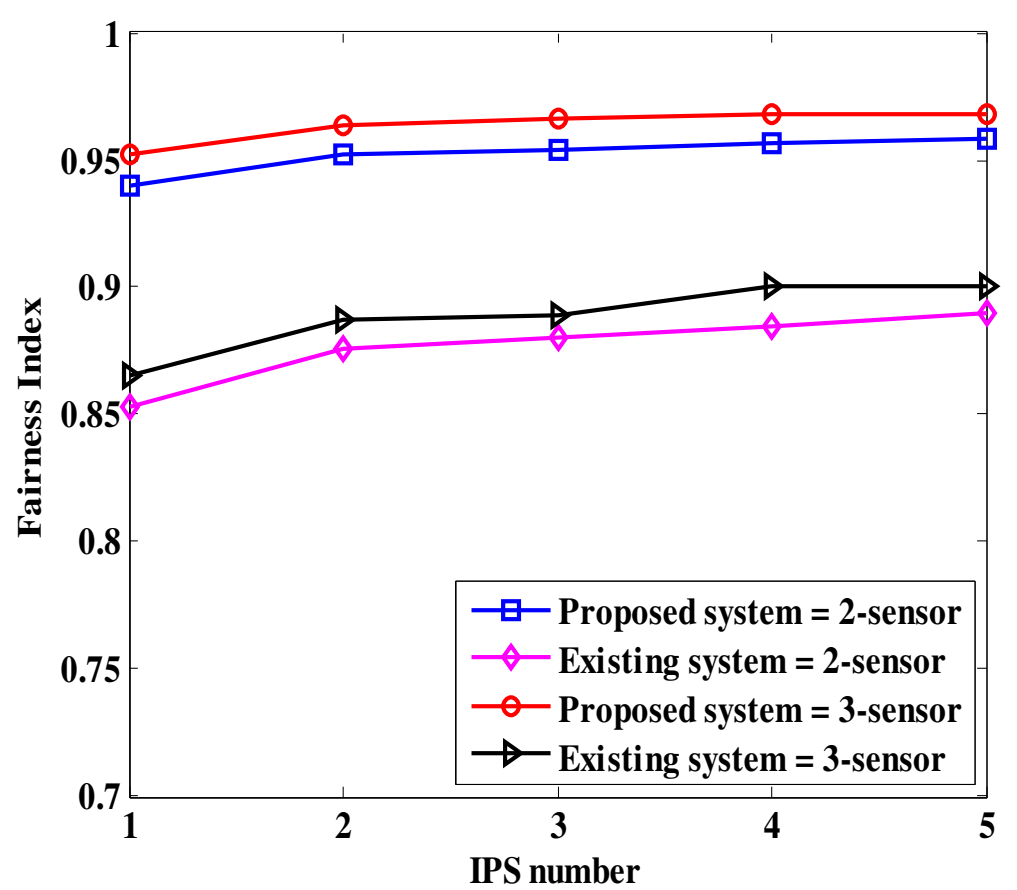

Fig. 7 System fairness against IPS number

proposed algorithm. The network distances to the BS are kept constant, while the number of IPSes is varied. To achieve this, we consider the deployment of class A to the BS at $5.5 \mathrm{~m}$, while class B is $6.5 \mathrm{~m}$ from the BS.
The two classes of network contain three sensors, powered by a number of intended three W IPS sources, which is varied in different simulation runs. As illustrated in Fig. 8, the results obtained are compared to the

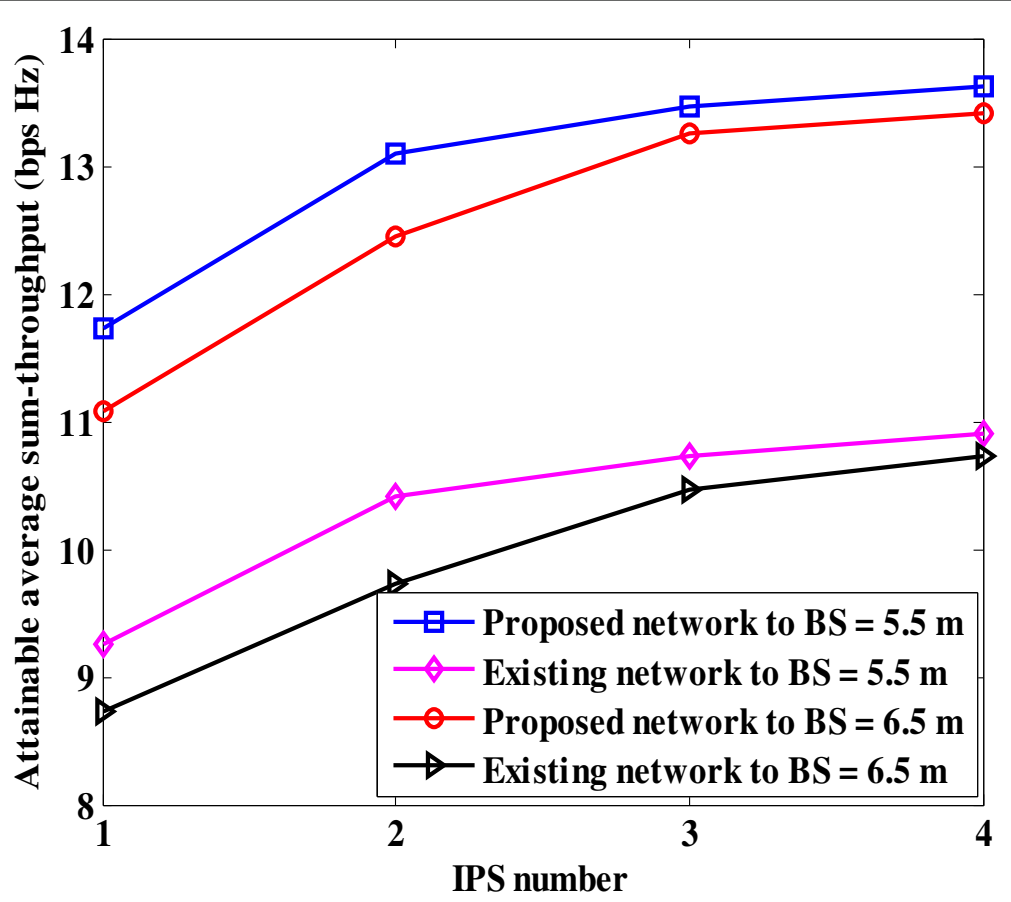

Fig. 8 Attainable average sum-throughput based on unequal network distance 
existing system with the same configuration, and it is apparent that a substantial increase of $25.68 \%$ and $26.67 \%$ in transmission throughput rate is attainable with the proposed system for class A and class B, particularly when the available energy is constrained by a small number of IPSes. Furthermore, class A in the proposed system, which has a smaller distance to the BS, must spend lower energy on information transmission to the $\mathrm{BS}$ in the UL, and consequently, the network achieved a significant improvement in the attainable average sum-throughput compared to class B.

\subsection{Transmission power impact on the attainable throughput of the system}

This section investigates the influence of transmission power on the attainable throughput rate of a system with three-sensor, powered by a variable number of IPSes. To achieve this, the transmission power of the IPS is varied from $100 \mathrm{~mW}, 500 \mathrm{~mW}, 1000 \mathrm{~mW}$, to $3000 \mathrm{~mW}$. As depicted in Fig. 9, a great surge in the performance of the system is noticed as the IPS transmission power increases. Based on this observation, it can be corroborated that the IPS plays a crucial role in the attainable system sum-throughput rate in the context of transmission power value.

Similarly, with a larger number of IPS, the attainable overall throughput of the system performance gets better as more resources are being efficiently allocated to the network sensors in an optimal fashion. Furthermore, when the system is operated with $100 \mathrm{~mW}$ transmission power, which is quite low, the system performance is satisfactory. This is an interesting observation that depicts the proposed system's capability to efficiently utilize energy resources, with reliable network communication.

\subsection{Comparison based on equal network distances to the BS}

The effect of equal network distance to the BS between class A and class B networks is investigated in this section. As a result, the distances of the network classes are fixed, while the number of IPSes is varied in the course of the experiment. Class A and class B were considered at an equal distance of $7.5 \mathrm{~m}$ to the BS. Each class contains two sensors and they are powered by a variable number of three W IPS sources. The same investigation was carried out for three sensors. As illustrated in Fig. 10, it is noticeable that class A network only has a slight enhanced attainable sum-throughput compared to class B network for two sensors, regardless of the unequal distances among the randomly placed class B sensors, while the attainable throughput rate for three sensors was almost similar for classes A and B networks. As a consequence, it is apparent that the proposed optimization algorithm is able to efficiently handle resource allocation among the two classes of network in a fair manner by providing different optimal timing to class B sensors

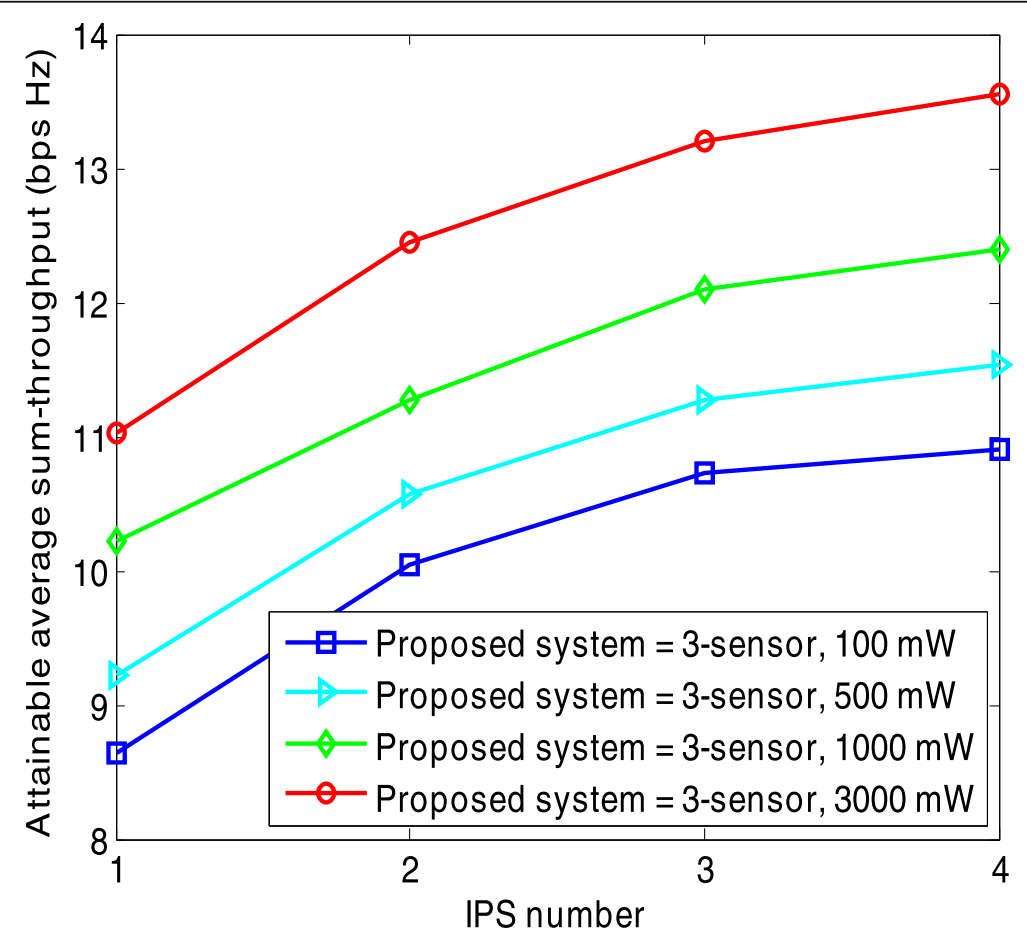

Fig. 9 Attainable average sum-throughput against transmission power 


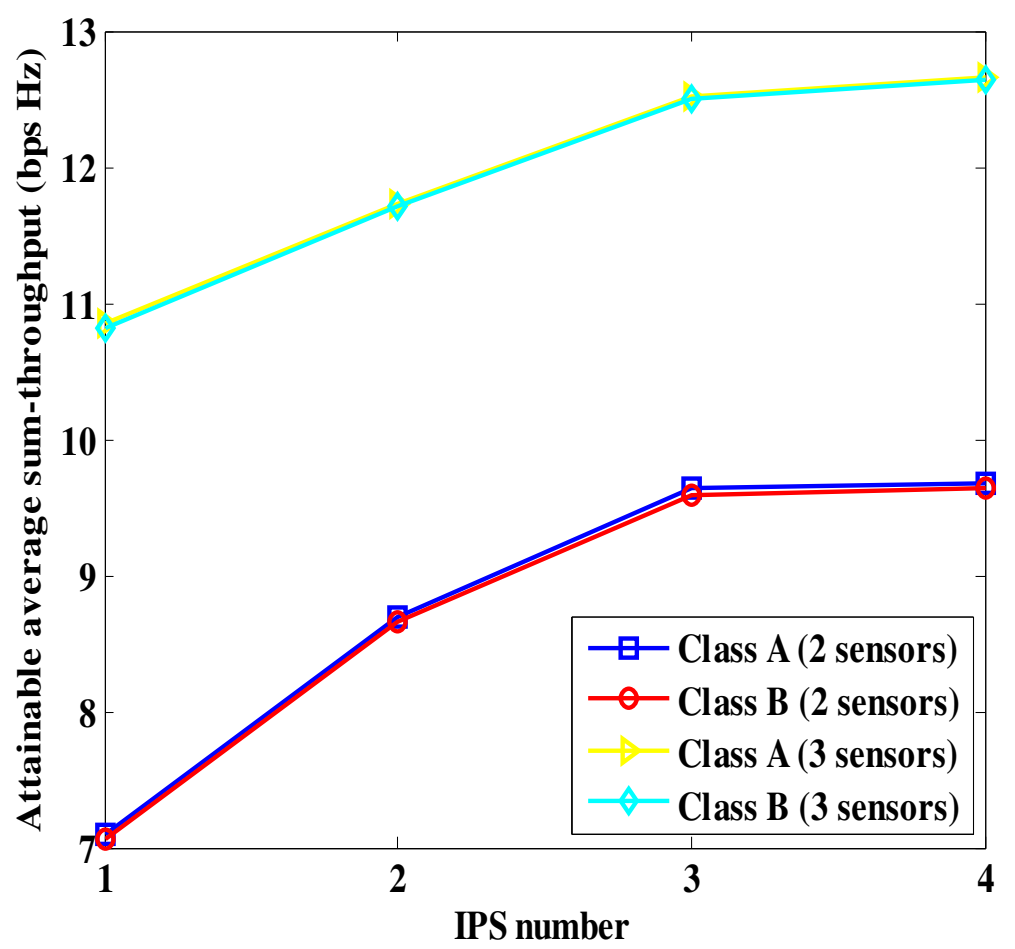

Fig. 10 Attainable average sum-throughput based on equal network distance

based on their calculated distances to the available energy resources, as well as equal optimal timing to the sensor nodes in class A.

\subsection{System performance based on fraction of energy consumed on information transmission}

This section investigates the performance of the system, using a three-sensor example, by varying the fraction of average power of transmission of the sensor nodes. Consequently, the number of both the network sensor nodes and the IPSes are fixed, as the average transmission power is varied. The system model is developed such that energy consumption for other networking processing has been optimized; therefore, it is possible to increase the amount of energy resources for information transmission. This is the basis for varying the fraction of average power of transmission.

From Fig. 11, it is noticeable that the system throughput rate increases as the information transmission power of the sensor nodes in the network increases, as could be expected. The reason for this is that the sensor nodes can now spend higher energy on information transmission during the UL period, which in turn improved the system overall throughput. In addition, the results obtained in Fig. 11 are compared to the existing system with the same configuration and it can be inferred that there is a significant improvement in the throughput rate of the proposed system. This is an indication that the proposed system is more energy-efficient in terms of energy consumption.

\subsection{Comparison of system performance under different number of nodes in the network classes}

In the section, the impact of different number of nodes in the network classes on the system performance is studied. To realize this, the number of nodes in class A and class B were unequal and constant, while number of IPSes was varied, in the course of simulation. Both classes $\mathrm{A}$ and $\mathrm{B}$ have an equal distance of $7.5 \mathrm{~m}$ to the BS. Comparison experiments were carried out on class A containing two sensors and class B containing three sensors; class A containing three sensors and class B containing four sensors; and class A containing two sensors and class B containing four sensors. Each experiment was powered by a variable number of three $\mathrm{W}$ IPS sources. From Fig. 12, it is noticeable that in all three the experiments, class B achieved a significant attainable throughput rate compared to class $\mathrm{A}$. The variation in the attainable throughput rates between class $A$ and class $B$ in the system is due to the number of sensor nodes contained in each network as the class with a higher number of nodes achieves a higher throughput rate compared to a class with a lesser number of nodes. 


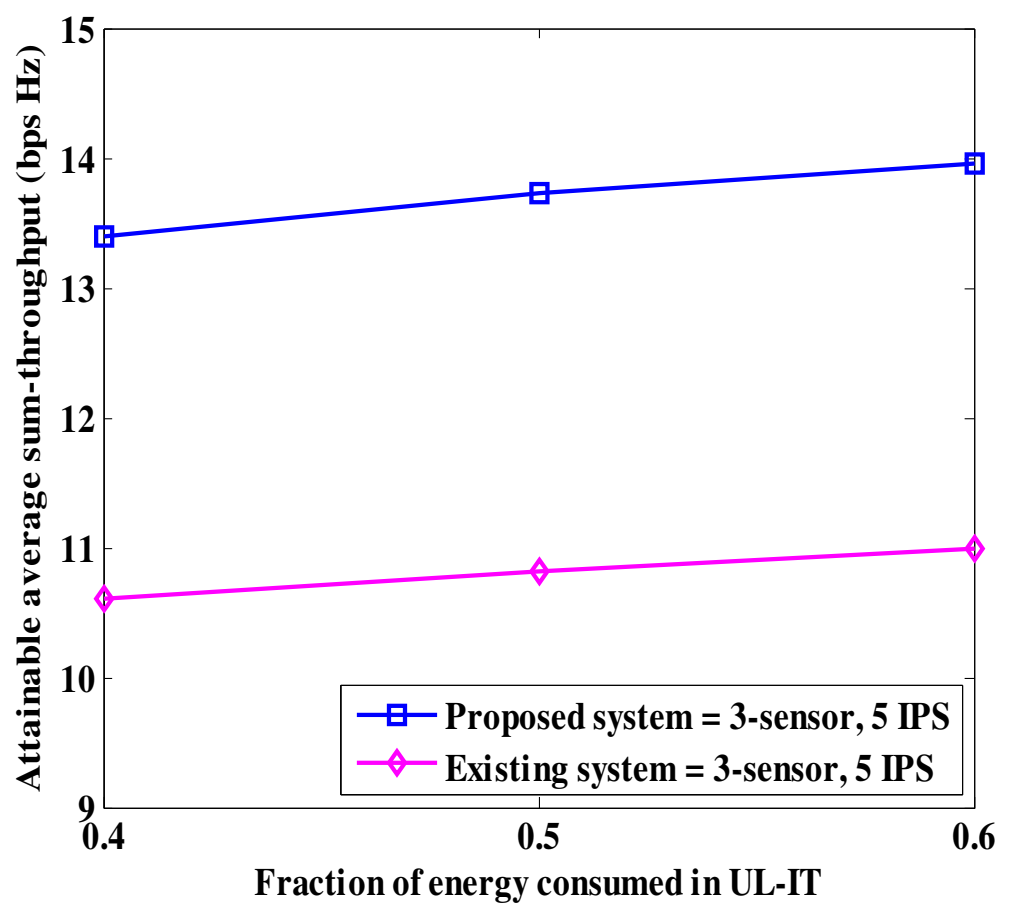

Fig. 11 Attainable average sum-throughput against fraction of energy consumed on information transmission

\section{Conclusion}

This paper has proposed a new approach to energy harvesting and data transmission optimization in a heterogeneous multi-class and multiple resource wireless transmission system that focuses on monitoring water and its quality. To achieve this, an optimal optimization algorithm that optimizes the energy and information timing schedules in a joint manner is proposed, and the proposed algorithm is validated in terms of path-loss exponent impact, performance comparison of systems, convergence based on iteration, comparison based on unequal network distances to the BS, transmission power impact on the attainable throughput and on the fraction of energy consumed on information transmissions, and influence of different number of nodes in the network classes. To reduce the system's energy consumption and to also enhance the system's overall throughput rate, a sum-throughput optimization technique is employed. The proposed system has revealed advantageous results in the context of fairness and sum-throughput by efficiently allocating resources to the deployed sensor nodes based on a determined strategy for one class, and a random strategy for the other. The new WPSN system was compared with an existing system in [36] based on transmission throughput rate and fairness. The transmission throughput rate and fairness results of a system with two-sensor employing the proposed algorithm performs comparably to a system with three-sensor employing an existing algorithm proposed in [36], with all other network parameters the same. In addition, the proposed optimization algorithm achieved a profitable transmission throughput rate regardless of the varying distances to the BS among the sensor nodes in class B of the proposed WPSN, when compared to a system with the same configuration, but without the proposed optimization algorithm. Moreover, for an IPS power as low as $100 \mathrm{~mW}$, the proposed system reveals an acceptable performance, which indicates it capability to efficiently utilize energy resources with reliable network communications.

\section{Appendix 1}

\subsection{Problem P1 transformation convexity proof}

The parameters of (21) such as $\log$ function, rendered the optimization problem as a non-convex function, and it was transformed through problem structure exploration technique by changing variable $t_{c} \xi_{0}$ to $\xi_{0, c}$, and obtaining the natural $\log$ of the $\log$ function in (9) and (18) respectively, to obtain a new problem (P2). From (9) and (18), (27) and (28) were derived as follows:

$$
R_{a}(j, \zeta)=\frac{\zeta_{a}}{\operatorname{In} 2} \operatorname{In}\left(1+\alpha_{a} \frac{\sum_{c=1}^{C} j_{c}}{\zeta_{a}}\right), \forall a=1,2, \ldots, \mathrm{A}
$$




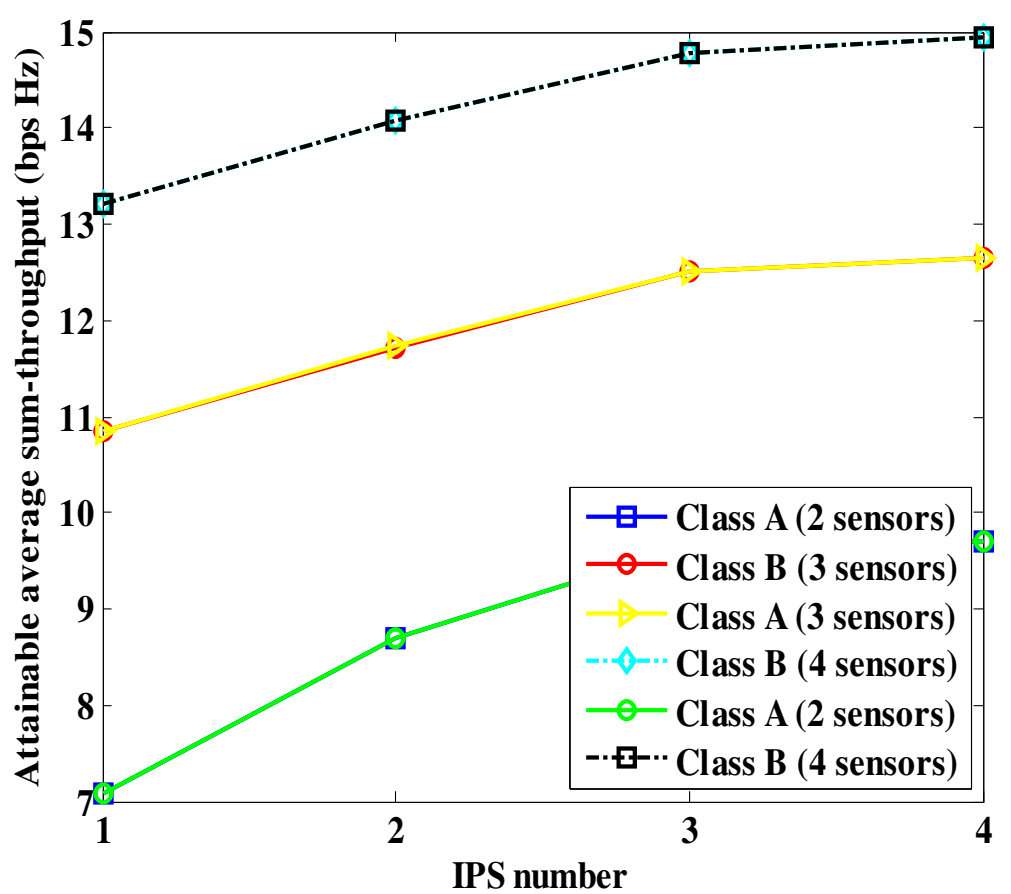

Fig. 12 Attainable sum-throughput based on different number of nodes in the network classes

$$
R_{b}(\xi)=\frac{\xi_{b}}{\operatorname{In} 2} \operatorname{In}\left(1+\gamma_{b} \frac{\sum_{c=1}^{C} \xi_{0, c}}{\xi_{b}}\right), \forall a=1,2, \ldots, \mathrm{B}
$$

where:

$$
j=j_{1}, j_{2}, \ldots, j_{C}, \zeta=\zeta_{1}, \zeta_{2}, \ldots, \zeta_{A}
$$

$$
\xi_{b} \geq 0, \forall b=1,2, . ., \mathrm{B}
$$

The concavity of problem (P2) is shown as follows through the application of partial derivative

$$
R_{a, b}(j, \zeta, \xi)=\frac{\zeta_{a}}{\operatorname{In} 2} \operatorname{In}\left(1+\alpha_{a} \frac{\sum_{c=1}^{C} j_{c}}{\zeta_{a}}\right)+\frac{\xi_{b}}{\operatorname{In} 2} \operatorname{In}\left(1+\gamma_{b} \frac{\sum_{c=1}^{C} \xi_{0, c}}{\xi_{b}}\right)
$$

and

$$
\xi=\xi_{0,1}, \xi_{0,2}, \ldots, \xi_{0, M}, \xi_{1}, \xi_{2}, \ldots, \xi_{B}
$$

The sum-throughput of the overall sensor nodes in class $\mathrm{A}$ and Class $\mathrm{B}$, which is defined by $R_{a, b}$ is:

$$
R_{a, b}(j, \zeta, \xi)=\sum_{a=1}^{A} R_{a}(j, \zeta)+\sum_{b=1}^{B} R_{b}(\xi)
$$

Consequently, the optimization problem (P1) is transformed to a new problem defined as (P2).

(P2):

$$
{ }_{j, \zeta, \xi} R_{a, b}(j, \zeta, \xi)
$$

s.t:

$$
\sum_{c=1}^{C} j_{c}+\sum_{c=1}^{C} \xi_{0, c}+\sum_{a=1}^{A} \zeta_{a}+\sum_{b=1}^{B} \xi_{b} \leq 1
$$

$j_{c} \geq 0, \forall c=1,2, . ., \mathrm{C}$

$\zeta_{a} \geq 0, \forall a=1,2, . ., \mathrm{A}$
The second derivative of $R_{a, b}(j, \zeta, \xi)$ and $j_{c}$ is:

$$
\frac{\partial^{2} R_{a, b}(j, \zeta, \xi)}{\partial j_{c}^{2}}=\frac{-\alpha_{a}^{2} \zeta_{a}}{\operatorname{In} 2\left(\zeta_{a}+\alpha_{a} \sum_{c=1}^{C} j_{c}\right)^{2}}
$$

Since $\alpha_{a}^{2}$, In 2 , and $\left(\zeta_{a}+\alpha_{a} \sum_{c=1}^{C} j_{c}\right)$ are positive, then,

$$
\frac{\partial^{2} R_{a, b}(j, \zeta, \xi)}{\partial j_{c}^{2}}=\frac{-\alpha_{a}^{2} \zeta_{a}}{\operatorname{In} 2\left(\zeta_{a}+\alpha_{a} \sum_{c=1}^{C} j_{c}\right)^{2}} \leq 0
$$

if $\zeta_{a} \geq 0$. Hence, $R_{a, b}(j, \zeta, \xi)$ is concave with respect to $j_{c}$.

The second derivative of $R_{a, b}(j, \zeta, \xi)$ vis-à-vis $\xi_{0, c}$ gives:

$$
\frac{\partial^{2} R_{a, b}(j, \zeta, \xi)}{\partial \xi_{0, c}{ }^{2}}=\frac{-\gamma_{b}^{2} \xi_{b}}{\operatorname{In} 2\left(\xi_{b}+\gamma_{b} \sum_{c=1}^{C} \xi_{0, c}\right)^{2}}
$$


Since $\gamma_{b}^{2}$, In 2 , and $\left(\xi_{b}+\gamma_{b} \sum_{c=1}^{C} \xi_{0, c}\right)$ are positive, then,

$$
\frac{\partial^{2} R_{a, b}(j, \zeta, \xi)}{\partial \xi_{0, c}^{2}}=\frac{-\gamma_{b}^{2} \xi_{b}}{\operatorname{In} 2\left(\xi_{b}+\gamma_{b} \sum_{c=1}^{C} \xi_{0, c}\right)^{2}} \leq 0
$$
$\xi_{0, c}$

if $\varepsilon_{b} \geq 0$. Hence, $R_{a, b}(j, \zeta, \xi)$ is concave with respect to The second derivative of $R_{a, b}(j, \zeta, \xi)$ and $\zeta_{a}$ gives:

$$
\frac{\partial^{2} R_{a, b}(j, \zeta, \xi)}{\partial \zeta_{a}^{2}}=\frac{-\alpha_{a}}{\zeta_{a}^{2} \mathrm{In} 2}\left(1+\alpha_{a} \frac{\sum_{c=1}^{C} j_{c}}{\zeta_{a}}\right)^{-2} \cdot\left(\sum_{c=1}^{C} j_{c}\right)^{2}
$$

Also, the second derivative of $R_{a, b}(j, \zeta, \xi)$ and $\xi_{b}$ gives:

$$
\begin{gathered}
\frac{\partial^{2} R_{a, b}(j, \zeta, \xi)}{\partial \xi_{b}{ }^{2}}=\frac{-\gamma_{b}}{\xi_{b}^{2} \operatorname{In} 2}\left(1+\gamma_{b} \frac{\sum_{c=1}^{C} \xi_{0, c}}{\xi_{b}}\right)^{-2} \cdot\left(\sum_{c=1}^{C} \xi_{0, c}\right)^{2} \\
\frac{\partial^{2} R_{a, b}(j, \zeta, \xi)}{\partial j_{c} \partial \zeta_{a}}=\frac{\alpha_{a}^{2}}{\zeta_{a}^{2} \operatorname{In} 2}\left(1+\alpha_{a} \frac{\sum_{c=1}^{C} j_{c}}{\zeta_{a}}\right)^{-2} \cdot \sum_{c=1}^{C} j_{c} \\
\frac{\partial^{2} R_{a, b}(j, \zeta, \xi)}{\partial \xi_{0, c} \partial \xi_{b}}=\frac{\gamma_{b}^{2}}{\xi_{b}^{2} \operatorname{In} 2}\left(1+\gamma_{b} \frac{\sum_{c=1}^{C} \xi_{0, c}}{\xi_{b}}\right)^{-2} \cdot \sum_{c=1}^{C} \xi_{0, c} \\
\frac{\partial^{2} R_{a, b}(j, \zeta, \xi)}{\partial j_{c} \partial \xi_{b}}=0 ; \frac{\partial^{2} R_{a, b}(j, \zeta, \xi)}{\partial \xi_{0, c} \partial \zeta_{a}} \\
=0 ; \frac{\partial^{2} R_{a, b}(j, \zeta, \xi)}{\partial \zeta_{a} \partial \xi_{b}}=0 ;
\end{gathered}
$$

From the second derivative test, $R_{a, b}(j, \zeta, \xi)$ is a concave function and it can be solved through any known standard convex method.

\section{Appendix 2}

\subsection{Minimization of energy harvesting unfairness}

Using (22a), that is:

$$
\sum_{c=1}^{C} j_{c}+\sum_{c=1}^{C} t_{c}=1
$$

$$
\begin{aligned}
& \text { If } \\
& \sum_{c=1}^{C} P_{c} n_{a} j_{c}+\sum_{c=1}^{C} P_{c} u_{b} t_{c} \xi_{0}=\sum_{c=1}^{C} P_{c} n_{a} j_{c}+\varepsilon_{0} \sum_{c=1}^{C} P_{c} u_{b} t_{c}
\end{aligned}
$$

Then, (45) is true.

By substituting (46) in (4) and (15), (47) is derived as:

$$
\begin{aligned}
E_{a, b} & =\varepsilon_{a} \sum_{c=1}^{C} P_{c} n_{a} j_{c}+\varepsilon_{b} \xi_{0} \sum_{c=1}^{C} P_{c} u_{b} t_{c}, a \\
& =1,2, \ldots, A, b=1,2, \ldots, B
\end{aligned}
$$

Let $E_{a, b}^{*}$ be the optimal value for $E_{a}$ and $E_{b}$ due to $j_{c}^{*}$, $t_{c}^{*}$, and $\xi_{0}^{*}$.

Since $j_{c}^{*}$ represents the optimal value for $j_{c}$, while $t_{c}^{*}$ and $\xi_{0}^{*}$ represents the optimal values for $t_{c}$ and $\xi_{0}$. Therefore, from (47), we have:

$$
\begin{aligned}
E_{a, b}^{*} & =\varepsilon_{a} \sum_{c=1}^{C} P_{c} n_{a} j_{c}^{*}+\varepsilon_{b} \xi_{0}^{*} \sum_{c=1}^{C} P_{c} u_{b} t_{c}^{*}, a \\
& =1,2, \ldots, A, b=1,2, \ldots, B
\end{aligned}
$$

From $(48), j_{c}^{*}$ remains constant. Similarly, $t_{c}^{*}$ remains constant regardless of $\xi_{0}^{*}$.

\section{Acknowledgments}

The authors are grateful to the anonymous reviewers for their insightful comments which have contributed to improving the quality of this paper. The authors would like to appreciate the support of Dr. B.S. Awoyemi. This work is based on the research supported by the National Research Foundation. Any opinion, finding and conclusion, or recommendation expressed in this material is that of the author(s) and the NRF does not accept any liability in this regard.

\section{Availability of data and materials}

The authors declare that the data and materials in this manuscript are available.

\section{Authors' contributions}

SO conceived and designed the study. SO also carried out the simulations and drafted the paper.TH contributed to the design and analysis of the study. TH was responsible for reviewing and editing of the paper. SO and $\mathrm{TH}$ read and approved the final version of the paper.

\section{Competing interests}

The authors declare that they have no competing of interests.

\section{Publisher's Note}

Springer Nature remains neutral with regard to jurisdictional claims in published maps and institutional affiliations.

Received: 25 August 2018 Accepted: 4 December 2018

Published online: 08 January 2019

\section{References}

1. K.C. Honeychurch, J.P. Hart, Screen-printed electrochemical sensors for monitoring metal pollutants. Trends Anal. Chem. 22(8), 456-469 (2003).

2. S.O. Olatinwo, T.H. Joubert, Energy efficient solutions in wireless sensor system for monitoring the quality of water: a review. IEEE Sensors J. https:// doi.org/10.1109/JSEN.2018.2882424.

3. J.P. Cabral, Water microbiology. Bacterial pathogens and water. Int. J. Environ. Res. Public Health 7(10), 3657-3703 (2010).

4. SM McLarnan, Escherichia coli as a water quality indicator organism: a case for responsive, science-based policy. All College Thesis Program. Available online: http://digitalcommons.csbsju.edu/honors_thesis/38 (accessed on 20 May 2018).

5. S.T. Odonkor, J.K. Ampofo, Escherichia coli as an indicator of bacteriological quality of water: an overview. Microbiol. Res. 4(1), 5-11 (2013).

6. Y. Li, Y. Wang, M. Cong, H. Lang, in Proceedings of the IEEE International Conference on Industrial Technology. Design and development of a water quality monitoring network and system (IEEE, Toronto, ON, Canada, 2017), pp. 912-916.

7. M. Pule, A. Yahya, J. Chuma, Wireless sensor networks: a survey on monitoring water quality. J Appl. Res. Technol. 15, 562-570 (2018). 
8. P.W. Alexander, L.T. Benedetto, T. Dimitrakopoulos, D.B. Hibbert, J.C. Ngila, M. Sequeira, D. D Shiels, Field-portable flow-injection analysers for monitoring of air and water pollution. Talanta 43(6), 915-925 (1996).

9. L. Pujol, D. Evrard, K. Groenen-Serrano, M. Freyssinier, A. Ruffien-Cizsak, P. Gros, Electrochemical sensors and devices for heavy metals assay in water: the French groups' contribution. Frontiers in Chemistry, 2, 1-23 (2014).

10. N. Khatri, S. Tyagi, Influences of natural and anthropogenic factors on surface and groundwater quality in rural and urban areas. Front. Life Sci. 8(1), 23-29 (2015)

11. A.A. Ensafi, K. Zarei, Simultaneous determination of trace amounts of cadmium, nickel and cobalt in water samples by adsorptive voltammetry using ammonium 2-amino-cyclopentene dithiocarboxylate as a chelating agent. Talanta 52(3), 435-440 (2000).

12. V. Somerset, J. Leaner, R. Mason, E. Iwuoha, A. Morrin, Development and application of a poly (2,2'-dithiodianiline) (PDTDA)-coated screen-printed carbon electrode in inorganic mercury determination. Electrochim. Acta 55(14), 4240-4246 (2010).

13. A. Fenwick, Waterborne infectious diseases - could they be consigned to history? Science 313(5790), 1077-1081 (2006).

14. K.C. Honeychurch, D.M. Hawkins, J.P. Hart, D.C. Cowell, Voltammetric behaviour and trace determination of copper at a mercury-free screenprinted carbon electrode. ELSEVIER Talanta 57(3), 565-574 (2002).

15. Y.I. Tur'yan, O.Y. Berezin, I. Kuselman, A. Shenhar, pH-metric determination of acid values in vegetable oils without titration. J. Am. Oil Chem. Soc. 73(3), 295-301 (1996).

16. D. Ozdemir, B. Ozturk, Near infrared spectroscopic determination of olive oil adulteration with sunflower and corn oil. J. Food Drug Anal. 15(1), 40-47 (2007).

17. N. Bahl, A.K. Sharma, H.K. Verma, On the energy utilization for WSN based on BPSK over the generalized-K shadowed fading channel. Wirel. Netw 20(8), 2385-2393 (2014).

18. W. Xu, W. Cheng, Y. Zhang, Q. Shi, X. Wang, On the optimization model for multi-hop information transmission and energy transfer in TDMA-based wireless sensor networks. IEEE Commun. Lett. 21(5), 1095-1098 (2017).

19. S.K. Nobar, K.A. Mehr, J.M. Niya, B.M. Tazehkand, Cognitive radio sensor network with green power beacon. IEEE Sensors J. 17(5), 1549-1561 (2017).

20. M. Shin, I. Joe, Energy management of algorithm for solar-powered energy harvesting wireless sensor node for internet of things. IET Commun. 10(12), 1508-1521 (2016).

21. S. Basagni, M.Y. Naderi, C. Petrioli, D. Spenza, in Mobile Ad Hoc Networking: The Cutting Edge Directions. Wireless sensor networks with energy harvesting (John Wiley \& Sons, New Jersey, USA, 2013), pp. 701-736.

22. X. Li, Q. Tang, C. Sun, Energy efficient dispatch strategy for the dualfunctional mobile sink in wireless rechargeable sensor networks. Wirel. Netw 24(3), 671-681 (2018).

23. F. Akhtar, M.H. Rehmani, Energy replenishment using renewable and traditional energy resources for sustainable wireless sensor networks: a survey. Renew. Sust. Energ. Rev. 45, 769-784 (2015).

24. S.B. Jeon, S. Kim, S.J. Park, M.L. Seol, D. Kim, Y.K. Chang, Y.K. Choi, Selfpowered electro-coagulation system driven by a wind energy harvesting triboelectric nanogenerator for decentralized water treatment. ELSEVIER Nano Energy 28, 288-295 (2016)

25. X. Lu, P. Wang, D. Niyato, D.I. Kim, Z. Han, Wireless networks with RF energy harvesting: a contemporary survey. IEEE Commun. Surv. Tutorials 17(2), $757-$ 789 (2015).

26. Powercast Co. (2017). Powercaster Transmitter, accessed on Sep. 7, 2017. [Online]. Available: http://www.powercastco.com/products/powercastertransmitter/.

27. Powercast Co. (2018). Powercaster Transmitter, accessed on Jan. 2, 2018. [Online]. Available: http://www.powercastsensors.com/products/wirelesstransmitters/.

28. Powercast Co. (2018). Market Wired, accessed on Jan. 2, 2018. [Online]. Available: http://www.marketwired.com/press-release/powercasts-wirelesspower-development-kit-with-fcc-approved-transmitter-charges-devices2221816.htm.

29. S. Bi, C.K. Ho, R. Zhang, Wireless powered communication: opportunities and challenges. IEEE Commun. Mag. 53(4), 117-125 (2015).

30. H. Tran, J. Akerberg, M. Bjorkman, HV Tran, RF energy harvesting: an analysis of wireless sensor networks for reliable. Wirel. Netw, 1-5 (2017). doi: https:// doi.org/10.1007/s11276-017-1546-6.
31. V. C. Gungor, G. P. Hancke (eds.), Industrial wireless sensor networks: Applications, protocols, and standards (Taylor \& Francis Crc Press, USA, 2013).

32. J. H, R. Zhang, Throughput maximization in wireless powered communication networks. IEEE Trans. Wirel. Commun. 13(1), 418-428 (2014).

33. Z. Gao, H.H. Chen, Y. Li, B. Vucetic, in Proceedings of the IEEE Australian Communications Theory Workshop, Melbourne. Wireless-powered communications with two-way information flow: protocols and throughput regions (VIC, Australia, 2016), pp. 29-34.

34. H. Ju, K. Chang, M.S. Lee, in Proceedings of the IEEE 17th International Conference on Advanced Communication Technology (ICACT). In-band fullduplex wireless powered communication networks (IEEE, Seoul, South Korea, 2015), pp. 23-27.

35. Z. Ling, F. Hu, L. Wang, J. Yu, X. Liu, Point-to-point wireless information and power transfer in WBAN with energy harvesting. IEEE Access 5, 8620-8628 (2017).

36. J.C. Kwan, A.O. Abraham, Radio frequency energy harvesting and data rate optimization in wireless information and power transfer sensor networks. IEEE Sensors J. 17(15), 4862-4874 (2017).

37. K. Chi, Y.H. Zhu, Y. Li, Efficient data collection in wireless powered communication networks with node throughput demands. Comput. Commun. 126, 1-10 (2018).

38. S.O. Olatinwo, T.H. Joubert, Optimizing the energy and throughput of a water-quality monitoring system. Sensors 18(4), 1198/1-21 (2018).

39. L. Mo, X. Cao, Y. Song, A. Kritikakou, Distributed node coordination for realtime energy-constrained control in wireless sensor and actuator networks. IEEE Internet Things J. 5(5), 4151-4163 (2018).

40. M. Shojafar, S. Abolfazli, H. Mostafaei, M. Singhal, Improving channel assignment in multi-radio wireless mesh networks with learning automata. Wirel. Pers. Commun. 82, 61-80 (2015).

41. K. Yamazaki, Y. Sugiyama, Y. Kawahara, S. Saruwatari, T. Watanabe, in Proceedings of the IEEE Wireless Communication Network Conference (WCNC). Preliminary evaluation of simultaneous data and power transmission in the same frequency channel (IEEE, New Orleans, LA, USA, 2015), pp. 1237-1242.

42. W. Ejaz, S. Kandeepan, A. Anpalagan, in Proceedings of the IEEE 26th Annual International Symposium on Personal, Indoor, Mobile Radio Communication (PIMRC). Optimal placement and number of energy transmitters in wireless sensor networks for RF energy transfer (IEEE, Hong Kong, 2015), pp. 1238-1243.

43. Texas Instruments. (2018). MSP430F1611 16-bit Uitra-Low Power MCU, accessed on Jan. 10, 2018. [Online].Available: http://www.ti.com/product/ MSP430F1611.

44. Powercast Co. (2018). Powercaster Receiver, accessed on Jan. 5, 2018. [Online]. Available: http://www.powercastco.com/products/powerharvesterreceivers/.

45. M. Carminati, V. Stefanelli, M. Sampietro, A. Turolla, M. Rossi, S. Malavasi, M. Antonelli, V. Pifferi, L. Falciola, in Proceedings of the IEEE Workshop on Environmental, Energy, and Structural Monitoring Systems (EESMS). Smart pipe: a miniaturized sensor platform for real-time monitoring of drinking water quality (2017), pp. 24-25.

46. F Regan, A Lawlor, A McCarthy, SmartCoast project - smart water quality monitoring system. Environmental Potection Agency, Ireland, pp. 1-35, [Online]. Available: https://www.epa.ie/pubs/reports/research/tech/STRIVE_ 30_Regan_SmartCoast_syn_web.pdf [accessed 2018-04-20] (2009).

47. CE Shannon, A mathematical theory of communication. ACM SIGMOBILE Mobile Computing and Communications Review, 5(1), 3-55 (2001).

48. S.P. Boyd, L. Vandenberghe, Convex Optimization (Cambridge University Press, Cambridge, U.K., 2009).

49. I. Flint, X. Lu, N. Privault, D. Niyato, P. Wang, Performance analysis of ambient RF energy harvesting with repulsive point process modeling. IEEE Trans. Wirel. Commun. 14(10), 5402-5416 (2015). 